\title{
Phase diagram of a two-site coupled complex SYK model
}

\author{
Antonio M. García-García, ${ }^{*}$ Jie Ping Zheng (郑杰平) ${ }^{\dagger}$ and Vaios Ziogas \\ Shanghai Center for Complex Physics, School of Physics and Astronomy, Shanghai Jiao Tong University, \\ Shanghai 200240, China
}

(Received 2 January 2021; accepted 19 April 2021; published 24 May 2021)

\begin{abstract}
We study the thermodynamic properties of a two-site coupled complex $q$-body Sachdev-Ye-Kitaev (SYK) model in the large- $N$ limit by solving the saddle-point Schwinger-Dyson (SD) equations. We find that its phase diagram is richer than in the Majorana case. In the grand canonical ensemble, we identify a region of small chemical potential and weak coupling between the two SYKs, for which two first order thermodynamic phase transitions occur as a function of temperature. First, we observe a transition from a cold wormhole phase to an intermediate phase that may correspond to a charged wormhole. For a higher temperature, there is another first order transition to the black hole phase. As in the Majorana case, the low temperature wormhole phase is gapped, and for sufficiently large coupling between the two complex SYK, or chemical potential, the first order transitions become crossovers. The total charge is a good indicator to study the phase diagram of the model; it is zero in the cold wormhole phase and jumps discontinuously at the temperatures at which the transitions take place. Based on the approximate conformal symmetry of the ground state, expected to be close to a thermofield double state, we identify the effective low energy action of the model. It is a generalized Schwarzian action with $S L(2, R) \times U(1)$ symmetry with an additional potential and an extra degree of freedom related to the charge. In the large $N$ and low temperature limit, results from this low energy action are consistent with those from the solution of the SD equations. Likewise, a large- $q$ analytical calculation of thermodynamic properties confirms the existence of the wormhole phase. Our findings are a preliminary step toward the characterization of traversable wormholes by its field theory dual, a charged strongly interacting fermionic system, that is easier to model experimentally.
\end{abstract}

DOI: 10.1103/PhysRevD.103.106023

\section{INTRODUCTION}

Traversable wormholes are classical solutions of Einstein's equations representing shortcuts in the geometry that may allow teletransportation among distant regions of space-time. For that reason, it has been a recurrent research theme for several decades [1-5]. Unfortunately, these solutions are considered classically unphysical as matter in these backgrounds would violate some fundamental physical principle, such as the null energy condition.

The situation changed recently [6], after it was shown that, turning on an interaction that couples the two boundaries of an eternal Bañados-Teitelboim-Zanelli black hole, the quantum matter stress tensor has a negative

\footnotetext{
*amgg@sjtu.edu.cn

jpzheng@sjtu.edu.cn

tvaios.ziogas@sjtu.edu.cn
}

Published by the American Physical Society under the terms of the Creative Commons Attribution 4.0 International license. Further distribution of this work must maintain attribution to the author(s) and the published article's title, journal citation, and DOI. Funded by SCOAP. average null energy without violating causality, which suggests that the wormhole becomes traversable. Other examples in different backgrounds and dimensionalities, but with similar features, were found shortly after [7-11]. The next main development came after Maldacena and Qi [12] (see also Ref. [13], in which the authors perform an explicit bulk time evolution) constructed a near two dimensional anti deSitter $\left(\mathrm{AdS}_{2}\right)$ background whose ground state was a time independent traversable wormhole, termed eternal traversable wormhole. Its highly entangled ground state is gapped and close to a thermofield double state (TFD) $[14,15]$. For sufficiently weak coupling, the system has a first order transition from the traversable wormhole phase to the (two) black hole phase. At a certain critical coupling, the gap vanishes, and the transition becomes a crossover. The boundary theory is given by a generalized Schwarzian action related to a modified Liouville quantum mechanical problem. Unlike the standard Sachdev-Y e-Kitaev (SYK) case [16,17], the spectrum for low energies is discrete, representing the wormhole phase. However, for higher energies, it is continuous, representing the black hole phase.

Interestingly, the field theory dual of this eternal traversable wormhole was identified [12] to be the low energy 
phase of a two-site coupled Sachdev-Ye-Kitaev model. It can be shown that the real time evolution of this model leads to the formation of a traversable wormhole [18]. Previously, a nonrandom SYK model $[19,20]$ was conjectured to describe similar physics. Indeed, both models share the same pattern of symmetry breaking $[21,22]$.

The SYK model [23-35] is a toy model for holography that, based on the same pattern of symmetry breaking from the full conformal group to $\operatorname{SL}(2, \mathrm{R})[36,37]$, is believed to be dual of a certain near $\mathrm{AdS}_{2}$ background [36]. Its main interest is that, despite being strongly interacting, and quantum chaotic [32], it is analytically tractable [32]. Distinctive features of the model include the saturation of a universal bound on chaos [38] typical of fast scramblers of information and systems with a gravity dual [38]; the exponential growth [39-41] of low energy excitations, typical of quantum black holes; and spectral correlations described by random matrix theory [40-44] that suggests that quantum dynamics is ergodic for sufficiently long times. The high temperature limit of the two-site SYK model [12], dual to two black hole backgrounds, shares most of these features. However, important differences arise in the low temperature limit corresponding to the wormhole phase. The system is no longer quantum chaotic. The low energy excitations are discrete even in the thermodynamics limit. This is consistent with the observation of a transition in level statistics [45], from integrable in the wormhole phase to quantum chaotic in the black hole region.

An appealing feature of the SYK model is that, at least potentially, it could be modeled experimentally [46-48]. That would allow not only the study of novel transport regimes in strongly interacting quantum dots but also, through holographic dualities, the experimental test of certain aspects of quantum gravity. However, this program is hampered by the difficulty to isolate and handle Majorana fermions.

In this paper, we study a generalization of the two-site SYK model with Majorana fermions, dual of the eternal traversable wormhole [12], to complex fermions with an extra $U(1)$ symmetry. More specifically, we shall study its expected field theory dual: a two-site coupled SYK model with Dirac, instead of Majorana, fermions. Single complex SYK have already been extensively investigated [49-53] in the literature. Qualitatively, they retain most of the interesting features of the Majorana SYK model while being closer to more realistic models of strongly interacting electrons. We shall see that, to some extent, this applies to the coupled charged SYK model. We shall find that the model is still gapped for low temperatures and weak coupling, which is a signature of the wormhole phase. For small chemical potential and low temperature, it has no charge, which is another feature of the traversable wormhole dual to the Majorana two-site SYK model [12]. Likewise, the high temperature phase is consistent with that of a system whose gravity dual is two black holes.
However, as we increase the chemical potential, we have identified qualitative differences between the Majorana and complex cases in the grand canonical ensemble. In a relatively narrow range of parameters, there exists an intermediate phase between the cold wormhole and black hole phase. For weak coupling, this intermediate phase is separated from the black hole and cold wormhole phases by two first order transitions. Tentatively, we believe that this novel phase may be a charged wormhole [18] characterized by a finite charge and a still gap in the spectrum. However, further research is required to confirm this point. For sufficiently large coupling, the transitions end in a crossover.

The paper is organized as follows. In Sec. II, we introduce the model, its expected ground state, and symmetries and derive the Schwinger-Dyson equations for the Green's functions in the large- $N$ limit. Based on the numerical solution of these equations, Sec. III is devoted to a detailed analysis of the thermodynamic properties of the model and the resulting phase diagram. Based on the approximate conformal symmetry of the ground state and its soft breaking in the low temperature limit, in Sec. IV, we write down the low energy generalized Schwarzian effective action and study some of its properties. This is the region of parameters where a gravity dual may exist. A list of problems for future research and conclusions are found in Sec. V.

\section{COUPLED COMPLEX SYK MODEL}

\section{A. Action}

The Hamiltonian of the complex SYK model is $[49,50]$

$$
H=\sum_{\{i\}} J_{i_{1} \ldots i_{q}} \psi^{i_{1} \dagger} \ldots \psi^{i_{q / 2} \dagger} \psi^{i_{q / 2+1}} \ldots \psi^{i_{q}},
$$

where $\{i\}=\left\{1 \leq i_{1}<i_{2}<\ldots<i_{q / 2} \leq N, 1 \leq i_{q / 2+1}<\right.$ $\left.\ldots<i_{q} \leq N\right\}$ and the complex coupling $J$ satisfies

$$
\begin{aligned}
J_{i_{1} \ldots i_{q / 2} i_{q / 2+1} \ldots i_{q}} & =J_{i_{q / 2+1} \ldots i_{q} i_{1} \ldots i_{q / 2}}^{*}, \\
J_{i_{1} i_{2} \ldots i i_{\frac{q}{2} ; i_{q}+1} \ldots i_{q}} & \left.=J_{\left[i_{1} i_{2} \ldots i_{q}\right] ; ; i i_{q}+1} \ldots i_{q}\right] \\
\overline{\left|J_{i_{1} \ldots i_{q}}\right|^{2}} & =\frac{(q / 2) !^{2}}{2 q N^{q-1}} J^{2},
\end{aligned}
$$

where the overbar denotes a statistical average with zero mean.

The Hamiltonian $H$ has a global $U(1)$ conserved charge

$$
Q=\frac{1}{N} \sum_{i} \psi^{i \dagger} \psi^{i}-\frac{1}{2}
$$

taking values in $(-1 / 2,1 / 2)$. We deform $H$ by adding a term $-N \mu Q$, where $\mu$ is the chemical potential of the $U(1)$ charge. 
In this paper, we consider two such SYKs with Dirac fermions, which we call "left" $L$ and "right" $R$. The two Hamiltonians $H_{L}$ and $H_{R}$ involve the same realization of the disordered coupling $J_{i_{1} \ldots i_{q}}$, and we set the chemical potentials to be equal, $\mu_{L}=\mu_{R}=\mu$, since we want to consider two identical copies of the theory. We couple the two systems using the interaction

$$
H_{\mathrm{int}}=-\frac{1}{2 N^{p-1}}\left[\sum_{i}\left(\eta \psi_{L}^{i \dagger} \psi_{R}^{i}+\eta^{*} \psi_{R}^{i \dagger} \psi_{L}^{i}\right)\right]^{p},
$$

where $\eta$ is in principle a complex coupling and $|\eta| \equiv \kappa{ }^{1}$ We choose $p=1$ for now, but it might also be interesting to consider $p>1$. Therefore, the total Hamiltonian is given by

$$
H_{\text {total }}=H_{L}+H_{R}+H_{\text {int }} \text {, }
$$

where $H_{R}=(-1)^{q / 2} H_{L}$.

\section{B. Symmetries}

We first discuss the symmetries of the uncoupled model $\eta=0$,

$$
H_{L}+H_{R}-N \mu\left(Q_{L}+Q_{R}\right) .
$$

There are two global symmetries $U(1)_{L}$ and $U(1)_{R}$, which can be combined as

$$
U(1)_{ \pm}=U(1)_{L} \pm U(1)_{R}
$$

where $U(1)_{+}$is interpreted as the symmetry of the system related to the total charge $Q_{+}=Q_{L}+Q_{R}$. Note that, due to the form of (6), $\mu$ can be thought of as a chemical potential for $Q_{+}$.

If we totally antisymmetrize the fermions in both left and right site, we also have a particle-hole symmetry at zero chemical potential $\mu=0[55,56]$,

$$
\psi_{a}^{i} \leftrightarrow \psi_{a}^{i \dagger}, \quad J_{i_{1} \ldots i_{q / 2} i_{q / 2+1} \ldots i_{q}} \rightarrow J_{i_{1} \ldots i_{q / 2} i_{q / 2+1} \ldots i_{q}}^{*},
$$

for $a=L, R$. The extra terms coming from the antisymmetrization are subleading in the $N$ expansion, so they do not affect our large- $N$ analytic arguments.

There is also an interchange symmetry,

$$
\psi_{L}^{i} \leftrightarrow \psi_{R}^{i}
$$

A general combination of $U(1)_{L} \times U(1)_{R}$ and the interchange symmetry (9) gives

\footnotetext{
${ }^{1}$ We can actually define $\eta$ with any phase if we perform a phase rotation in one of the systems, as explained in Ref. [54], but we keep it general in our analytical treatment.
}

$$
\psi_{L}^{i} \rightarrow e^{i \theta_{R}} \psi_{R}^{i}, \quad \psi_{R}^{i} \rightarrow e^{i \theta_{L}} \psi_{L}^{i} .
$$

The two-fermion coupling (4) breaks $U(1)_{-}$explicitly down to $\mathbb{Z}_{2}$, while it preserves $U(1)_{+} \cdot{ }^{2}$ Demanding that the combination (10) is preserved leads to the following relation between the fermionic phase in each site:

$$
\theta_{R}-\theta_{L}=2 \theta, \quad \theta \equiv \arg \eta .
$$

We make the convenient choice $\theta_{R}=-\theta_{L}=\theta$. $^{3}$

\section{Schwinger-Dyson equations}

One of our main goals is to study the thermodynamic properties of the system. For that purpose, the first step is to derive the saddle-point equations, termed SchwingerDyson (SD) equations [39], that control the large- $N$ limit of the Green's functions and self-energies that enter in the calculation of thermodynamic quantities. We first perform the statistical average of the path integral for the total action related to the Hamiltonian (5). To leading order in $N$, a straightforward calculation [16] yields

$$
\begin{aligned}
\langle Z\rangle_{J}= & \int D \psi_{a}^{i \dagger} D \psi_{a}^{i} \exp \left[-\sum_{i, a} \int \psi_{a}^{i \dagger}\left(\partial_{\tau}-\mu\right) \psi_{a}^{i}\right. \\
& +\sum_{i} \int\left(\eta \psi_{L}^{i \dagger} \psi_{R}^{i}+\eta^{*} \psi_{R}^{i \dagger} \psi_{L}^{i}\right)+\frac{(-1)^{q / 2}}{q N^{q-1}} J^{2} \\
& \left.\times \sum_{a, b} \iint s_{a b}\left(\left|\sum_{i} \psi_{a}^{i \dagger}(\tau) \psi_{b}^{i}\left(\tau^{\prime}\right)\right|^{2}\right)^{q / 2}\right],
\end{aligned}
$$

where $a=L, R$ and $s_{L L}=s_{R R}=1, s_{L R}=s_{R L}=(-1)^{q / 2}$.

We now define the Green's functions,

$$
G_{a b}\left(\tau, \tau^{\prime}\right)=\frac{1}{N} \sum_{i}\left\langle\mathcal{T} \psi_{a}^{i \dagger}(\tau) \psi_{b}^{i}\left(\tau^{\prime}\right)\right\rangle
$$

obeying

$$
G_{a b}^{*}\left(\tau, \tau^{\prime}\right)=G_{b a}\left(\tau, \tau^{\prime}\right) .
$$

The interchange symmetry (10), (11) implies that

$$
G_{L R}\left(\tau, \tau^{\prime}\right)=e^{-2 i \theta} G_{R L}\left(\tau, \tau^{\prime}\right),
$$

or $G_{L R}=-G_{R L}$ when $\theta=\pi / 2$.

The particle-hole symmetry (8) implies that

$$
G_{a b}\left(\tau, \tau^{\prime}\right)=-G_{b a}\left(\tau, \tau^{\prime}\right)
$$

\footnotetext{
${ }^{2}$ In Refs. [22,54], the $U(1)_{-}$was broken spontaneously by a quartic coupling, and Ref. [22] interpreted it as corresponding to a bulk gauge field in the gravity dual theory.

${ }^{3}$ Alternatively, Eq. (11) leads to the $\mathbb{Z}_{4}$ symmetry $\psi_{L}^{i} \rightarrow \psi_{R}^{i}$, $\psi_{R}^{i} \rightarrow-\psi_{L}^{i}$ being preserved upon choosing $\theta_{R}=0, \theta_{L}=\pi$, and $\eta$ purely imaginary as in Ref. [54].
} 
at zero chemical potential $\mu=0$. Combining (16) with (14) gives

$$
\begin{aligned}
& \operatorname{Re} G_{L L}=\operatorname{Re} G_{R R}, \quad \operatorname{Im} G_{L R}=-\operatorname{Im} G_{R L}, \\
& \operatorname{Im} G_{L L}=\operatorname{Im} G_{R R}=\operatorname{Re} G_{L R}=\operatorname{Re} G_{R L}=0 .
\end{aligned}
$$

This is for reference only; we do not assume these symmetries in the following as we want to work at $\mu \neq 0$.

At finite temperature $T=1 / \beta$, the Kubo-MartinSchwinger (KMS) condition (for the thermofield double state) reads [57]

$$
G_{a b}(\tau)=-G_{a b}(\tau+\beta), \quad \tau<0 .
$$

Introducing in the path integral (12) the Lagrange multipliers $\Sigma_{a b}\left(\tau, \tau^{\prime}\right)$ which enforce the definition (13), and integrating out the fermions assuming a replicadiagonal ansatz, we find

$$
\langle Z\rangle_{J} \sim \int D G D \Sigma \exp \left[-N I_{\mathrm{eff}}\right]
$$

where

$$
\begin{aligned}
-I_{\mathrm{eff}}= & \log \operatorname{det}\left[\delta_{a b}\left(\partial_{\tau}-\mu\right)+\Sigma_{a b}\right]+\int\left[\eta G_{L R}(\tau, \tau)+\eta^{*} G_{R L}(\tau, \tau)\right] \\
& +\sum_{a, b} \iint\left[\Sigma_{b a}\left(\tau^{\prime}, \tau\right) G_{a b}\left(\tau, \tau^{\prime}\right)+(-1)^{q / 2} q^{-1} J^{2} s_{a b}\left[G_{a b}\left(\tau, \tau^{\prime}\right)\right]^{q / 2}\left[G_{b a}\left(\tau^{\prime}, \tau\right)\right]^{q / 2}\right] .
\end{aligned}
$$

Varying the effective action (20) with respect to $G_{a b}$ and $\Sigma_{a b}$ leads to the following saddle-point SD equations,

$$
\begin{aligned}
\tilde{\Sigma}_{L L}\left(\tau, \tau^{\prime}\right)= & -(-1)^{q / 2} J^{2}\left[G_{L L}\left(\tau, \tau^{\prime}\right)\right]^{q / 2}\left[G_{L L}\left(\tau^{\prime}, \tau\right)\right]^{q / 2-1}+\left(\partial_{\tau}-\mu\right) \delta\left(\tau-\tau^{\prime}\right), \\
\tilde{\Sigma}_{L R}\left(\tau, \tau^{\prime}\right)= & -(-1)^{q} J^{2}\left[G_{L R}\left(\tau, \tau^{\prime}\right)\right]^{q / 2}\left[G_{R L}\left(\tau^{\prime}, \tau\right)\right]^{q / 2-1}+\eta \delta\left(\tau-\tau^{\prime}\right), \\
& \tilde{\Sigma}_{L L} \star G_{L L}\left(\tau, \tau^{\prime}\right)+\tilde{\Sigma}_{L R} \star G_{R L}\left(\tau, \tau^{\prime}\right)=-\delta\left(\tau-\tau^{\prime}\right), \\
& \tilde{\Sigma}_{L L} G_{L R}\left(\tau, \tau^{\prime}\right)+\tilde{\Sigma}_{L R} \star G_{R R}\left(\tau, \tau^{\prime}\right)=0,
\end{aligned}
$$

where the star $\star$ denotes the convolution $(f \star g)\left(\tau_{1}, \tau_{2}\right) \equiv \int d \tau f\left(\tau_{1}, \tau\right) g\left(\tau, \tau_{2}\right)$ and we have defined

$$
\tilde{\Sigma}_{a b}\left(\tau, \tau^{\prime}\right)=\Sigma_{a b}\left(\tau, \tau^{\prime}\right)+\left(\partial_{\tau}-\mu\right) \delta\left(\tau-\tau^{\prime}\right) \delta_{a b}
$$

We also get another set of equations by exchanging $L \leftrightarrow R$ and $\eta \leftrightarrow \eta^{*}$ in (21). For convenience, in the numerical calculation of thermodynamic properties, we set $\eta=-i \kappa$ and $q=4$.

These equations (21) are the analogous to those for the two-site coupled Majorana SYK model [12,18] whose gravity dual in the limit of low temperature and weak coupling between the left and right sites is the eternal traversable wormhole.

\section{Grand potential}

In the large- $N$ limit, the grand potential, computed by inserting the solution of the SD equations (21) in the on-shell action (20), is given by

$$
\begin{aligned}
-\frac{\beta \Omega}{N}= & 2 \ln \left[2 \cosh \frac{\beta \mu}{2}\right]+\operatorname{Tr} \ln \frac{\left(i \omega+\mu-\Sigma_{L L}\right)\left(i \omega+\mu-\Sigma_{R R}\right)-\Sigma_{L R} \Sigma_{R L}}{(i \omega+\mu)(i \omega+\mu)} \\
& -(-1)^{q / 2}\left(1-\frac{1}{q}\right) \sum_{a b} \int d \tau\left(J^{2} G_{a b}(\tau)^{q / 2} G_{b a}(\beta-\tau)^{q / 2}\right),
\end{aligned}
$$

where we have regularized the determinant as in Ref. [56]. From this expression, we can compute the rest of the thermodynamic quantities.

\section{THERMODYNAMIC PROPERTIES IN THE LARGE- $N$ LIMIT AND PHASE DIAGRAM}

In this section, we investigate the thermodynamic properties of the Hamiltonian (5) in the grand canonical ensemble, which will result in a detailed phase diagram of the model as a function of the coupling $\kappa$ between the two complex SYKs and the chemical potential $\mu$. We obtain all the thermodynamic quantities of interest by solving numerically the SD equations (21) using standard iterative techniques.

We will be mostly interested in the calculation of the total charge $Q$, related to the global $U(1)$ symmetry mentioned earlier and the grand potential $\Omega(T, \kappa, \mu)$ (23) where 


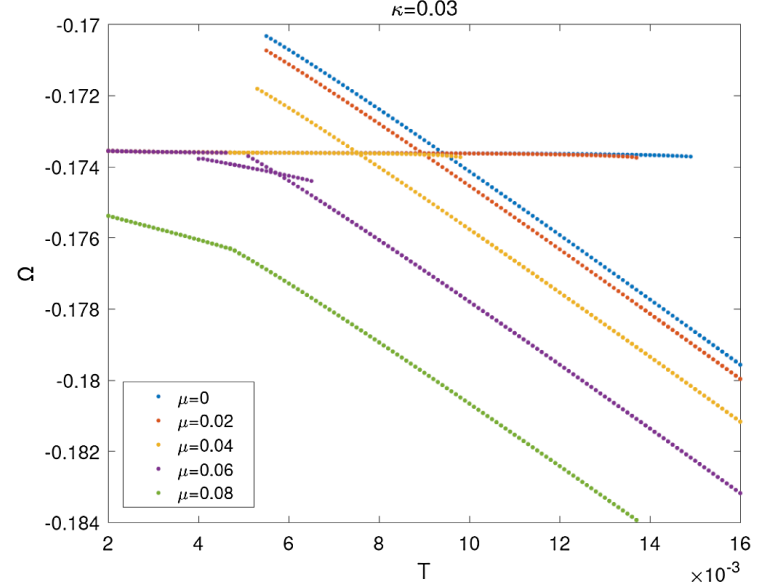

(a)

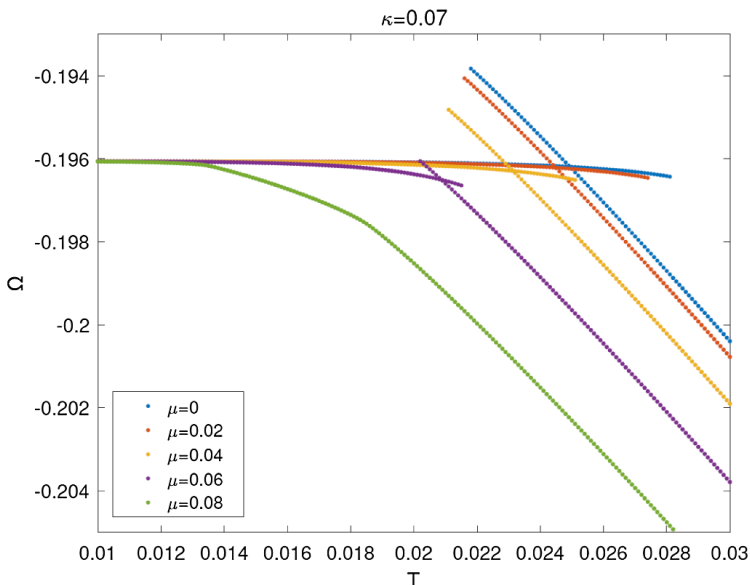

(c)

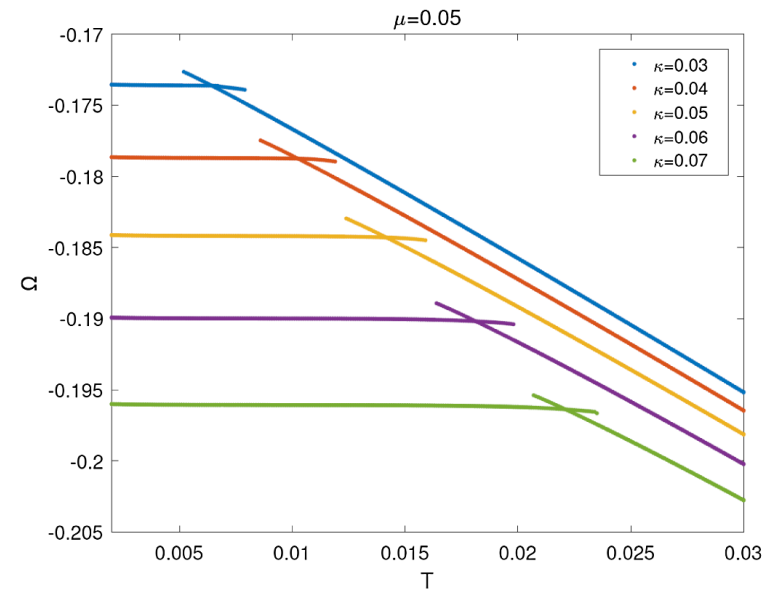

(e)

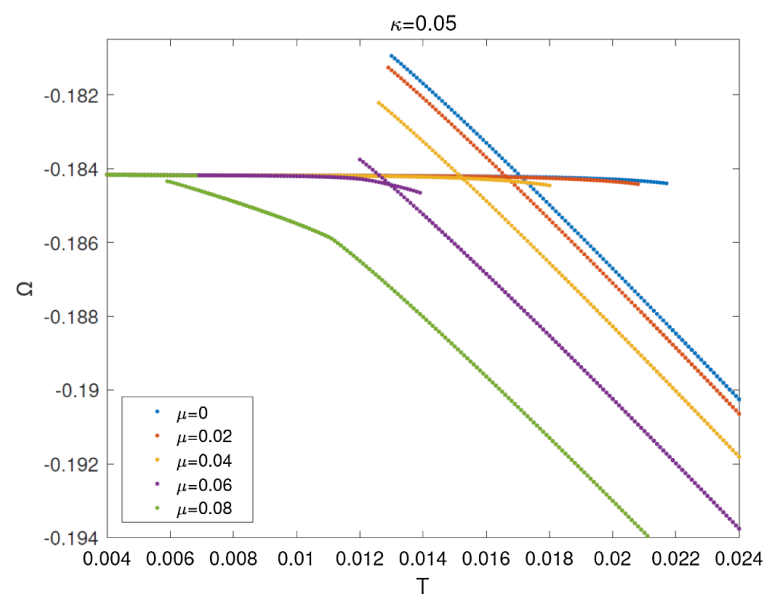

(b)

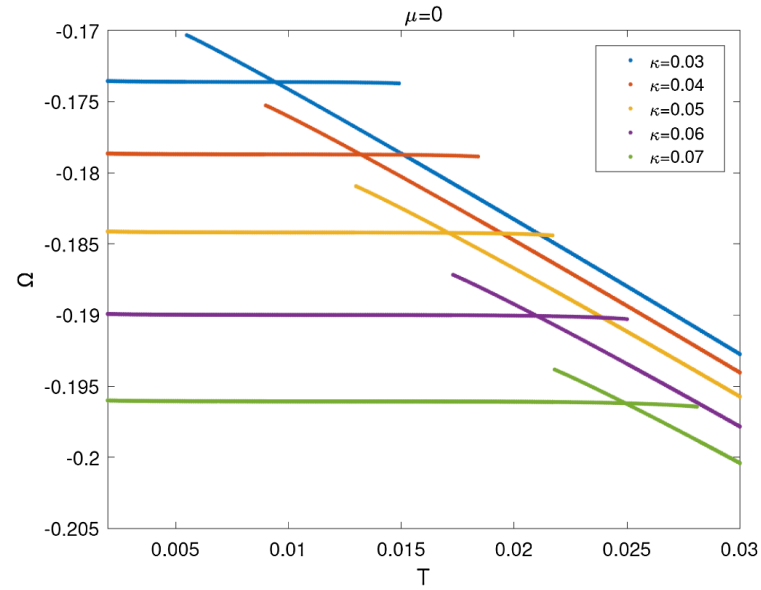

(d)

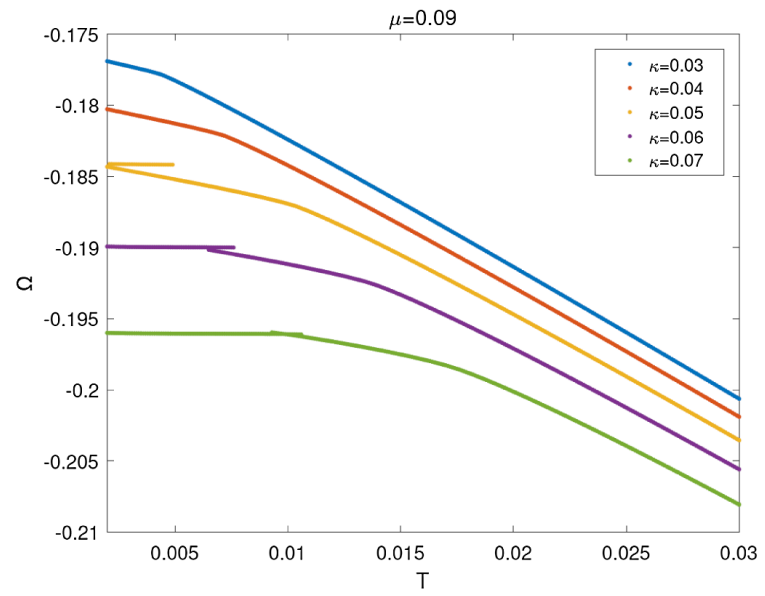

(f)

FIG. 1. Grand potential $\Omega$ vs temperature $T$ for $\kappa=0.03$ (a), $\kappa=0.05$ (b), $\kappa=0.07$ (c), with $\mu=0,0.02,0.04,0.06,0.08$ and for $\mu=0$ (d), $\mu=0.05$ (e), $\mu=0.09$ (f), with $\kappa=0.03,0.04,0.05,0.06,0.07$. The almost constant grand potential is a signature of the wormhole phase. A finite $\mu$ suppresses the wormhole phase and eventually induces a new phase transition for sufficiently low temperatures. As was expected, the increase of $\kappa$, for a fixed $\mu$, enhances the wormhole phase. 
$\kappa=|\eta|$. We shall see that these quantities characterize the different phases of the model and are easily accessible from the knowledge of the Green's functions and self-energies resulting from the solution of the SD equations (21). Likewise, the analysis of the exponential decay of $G_{L R}$ will provide useful information on the gap $E_{g}$ that characterizes the wormhole phase. We initiate our analysis with the calculation of the grand potential $\Omega$ (23).

\section{A. Grand potential $\boldsymbol{\Omega}(\boldsymbol{T}, \boldsymbol{\kappa}, \boldsymbol{\mu})$}

We compute the grand potential $\Omega$ as a function of the temperature $T$ for various $\kappa$ and $\mu$ by plugging in the action the Green's function and self-energies obtained by the numerical solution of the SD equations (21). The final expression for the grand potential, after a determinant regularization [51], is given by (23).

In general, the SD equations for a given temperature can have more than one solution corresponding to different phases of the model. The preferred solution is the one with a lower value of the grand potential.

Figure 1 depicts the temperature dependence of the grand potential for several couplings $\kappa$ (upper plots) and chemical potentials $\mu$ (lower plots). For $\mu=0$, results are very similar to the Majorana case [12]. For very low temperatures, and finite but small $\kappa$, the grand potential is almost temperature independent, suggesting the existence of a gap in the spectrum. This is the expected behavior in the traversable wormhole phase where the ground state, approximately described by a zero entropy TFD state, is separated from the first excited by a energy gap. As temperature increases, we observe a kink indicating a first order transition. In the proximity of the transition, we show the two branches of the grand potential. The gravity dual of the higher temperature phase is expected to be a two black hole geometry with an explicit coupling between the two backgrounds. For sufficiently strong coupling $\kappa \geq 0.125$, the first order transition ends. It is replaced by a smooth crossover of no evident gravitational interpretation.

The situation becomes more interesting for finite but small $\mu$. For sufficiently small $\mu$, the grand potential is similar to the $\mu=0$ case. We still observe a flat low temperature grand potential, related to a gap in the spectrum typical of the wormhole phase, that eventually ends in the first order transition mentioned above. The only difference is that $\mu$ slightly lowers both the gap and the critical temperature. This is expected as physically the chemical potential effectively increases the energy of the system that is detrimental of the gap in the wormhole phase which will vanish at a lower temperature.

However, for $\mu \sim 0.05$, we start to observe a second transition around $\kappa=0.05$. More specifically, as temperature increases, the wormhole phase undergoes a first order transition to an intermediate phase. At a higher temperature, another first order transition occurs from this intermediate phase to the black hole phase. That the

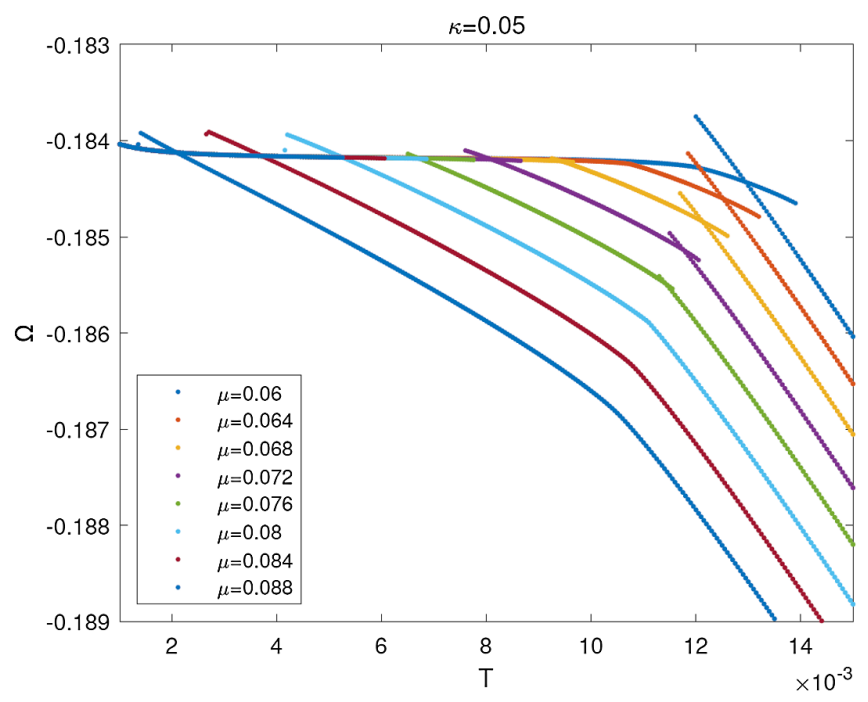

FIG. 2. Grand potential $\Omega$ vs temperature $T$ in the region of parameters $\kappa=0.05$ with $\mu=0.06, \ldots, 0.088$ where the two first order phase transitions are more clearly observed. We observe that the transitions become crossovers for sufficiently large $\mu$.

transition to the two black hole phase is the one at higher temperature can be inferred from the slope of the grand potential which is very similar for all values of the chemical potential no matter whether the intermediate phase exists or not. When $\mu$ increases further, this novel transition becomes also a crossover, so we can identify regions with two transitions, two crossovers, and one transition and one crossover. In general, the window of parameters where the two transitions are observed is rather narrow.

In Fig. 2, we choose the optimal choice of parameters for which this second transition is more clearly observed. We note that, despite the second transition occurring in a relatively small range of parameters, the region of coexistence of the different phases, given by the range of temperatures in which other branches are present, is still much smaller than the range of temperatures in which the intermediate phase occurs. This is a strong indication that this phase is stable in the grand canonical ensemble.

A technical comment is in order. As was mentioned earlier, the different solutions of the SD equations only exist in a determined range of temperatures. As the end points of each branch are approximated, the numerical calculation becomes increasing unstable with larger convergence time. We cannot rule out that a given branch survives for a somehow larger range of temperatures.

\section{B. Energy gap $E_{g}$ and the charge $Q$}

In order to further elucidate the phase diagram of the model, especially the nature of the intermediate phase, we study the energy gap and the charge $Q$ related to the global $U(1)$ symmetry mentioned in previous sections. We start with a detailed introduction of both concepts. 


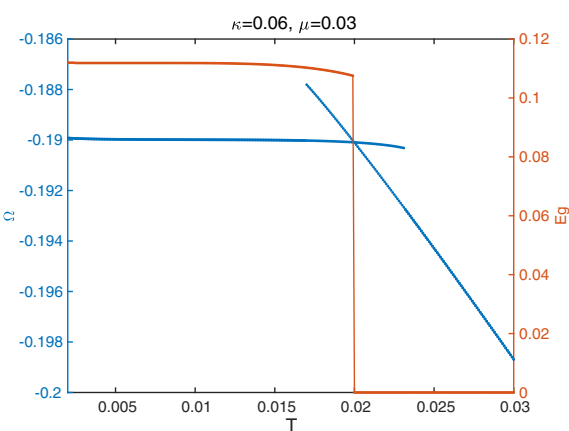

(a)

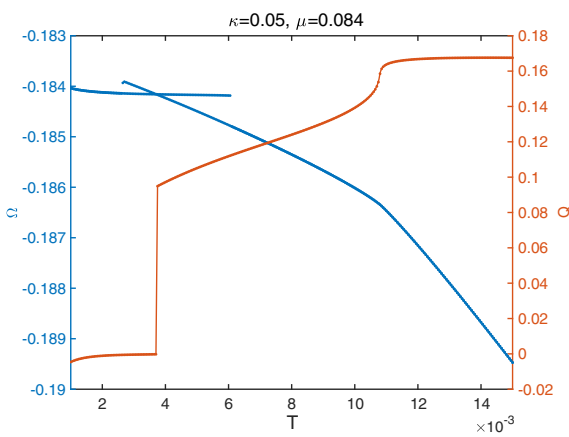

(d)

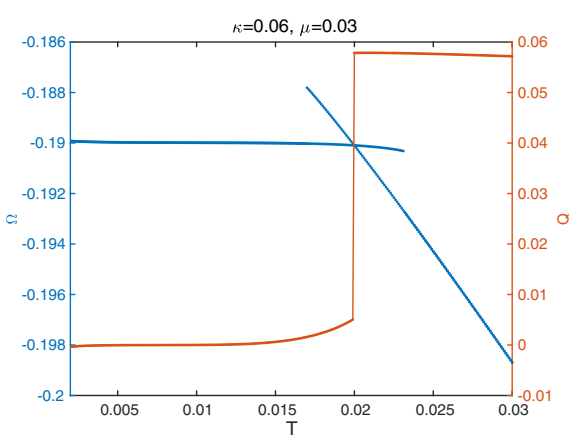

(b)

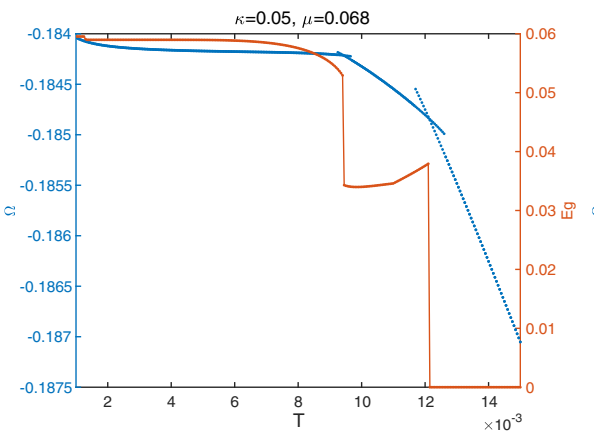

(e)

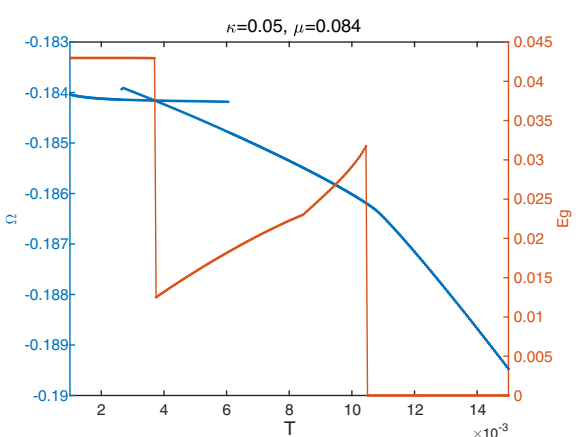

(c)

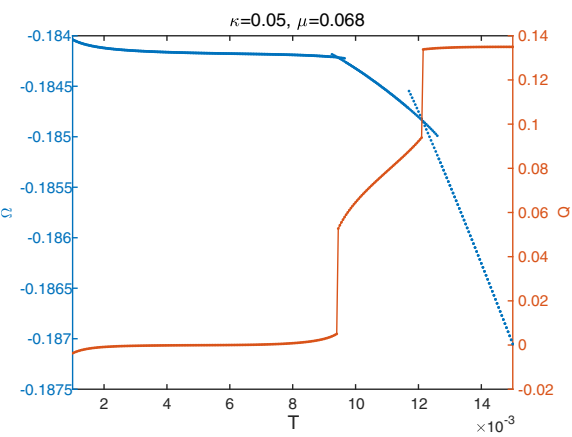

(f)

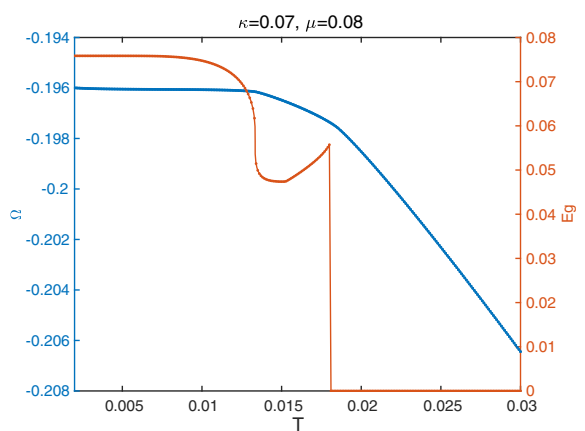

(g)

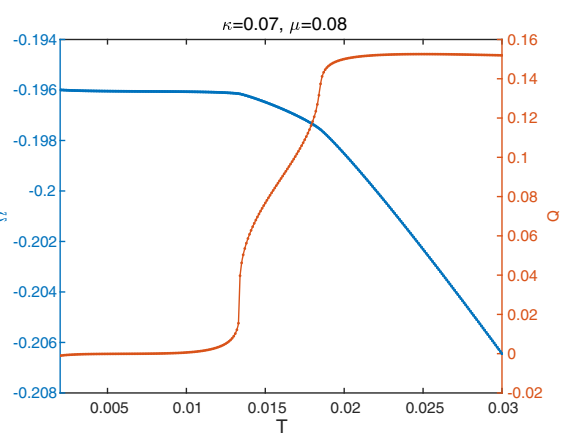

(h)

FIG. 3. $\Omega(T)$ and $E_{g}(T)$ (left column) and $\Omega(T)$ and $Q(T)$ (right column) for different $\mu$, $\kappa$. (a),(b) Only one phase transition, wormhole to black hole, is observed. (c),(d) Only the lower temperature transition is observed, and the high temperature one becomes a crossover. The fit to obtain the gap deteriorates rapidly for temperatures above the low temperature transition, so results are less reliable. (e),(f) Two phase transitions are observed. The fit to obtain the gap in the intermediate region is less reliable. (g),(h) For sufficiently large $\mu, \kappa$, both transitions become crossovers.

\section{Charge $Q$}

Following (3), we define the charge,

$$
Q_{a a}=\frac{1}{N} \sum_{i}^{N} \psi_{a i}^{\dagger} \psi_{a i}-\frac{1}{2}
$$

where the index $a=L, R$. Recalling the definition of the Green's function above,

$$
G_{a b}(\tau)=\frac{1}{N} \sum_{i}^{N}\left\langle T \psi_{a i}^{\dagger}(\tau) \psi_{b i}(0)\right\rangle \Rightarrow\left\{\begin{array}{l}
G_{a b}(\epsilon)=\frac{1}{N} \sum_{i}^{N}\left\langle\psi_{a i}^{\dagger}(\epsilon) \psi_{b i}(0)\right\rangle \\
G_{a b}(-\epsilon)=-\frac{1}{N} \sum_{i}^{N}\left\langle\psi_{b i}(0) \psi_{a i}^{\dagger}(-\epsilon)\right\rangle
\end{array}\right.
$$



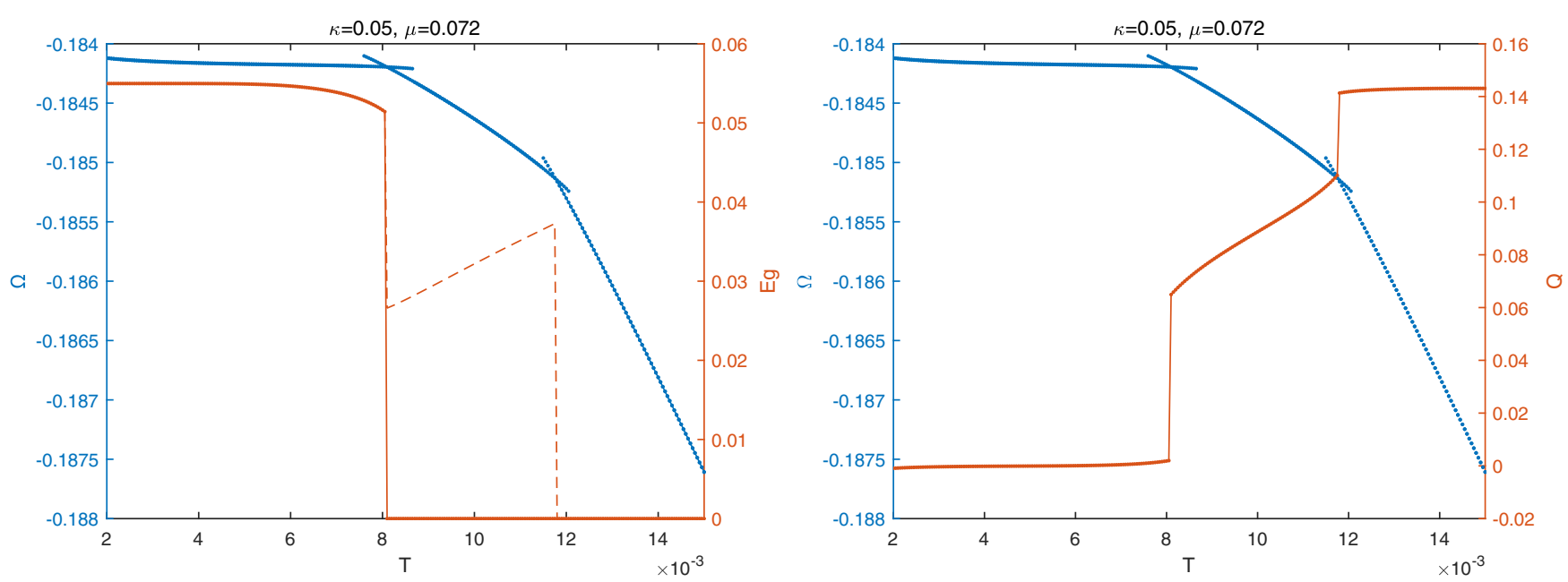

FIG. 4. Left: $\Omega(T)$ and $E_{g}(T)$ for $\kappa=0.05$ and $\mu=0.072$ where the two transitions are clearly observed. The solid line is the gap obtained by a linear fit of $\ln G_{L R}$. The dashed line is a similar fit, but the exponential decay of $G_{L R}$ occurs in a shorter interval, so the fitting is less reliable. Right: $\Omega(T)$ and $Q(T)$. In the wormhole low temperature phase, charge is zero. It jumps at both the low temperature and high temperature transitions. It increases linearly between the two transitions. This linear increase, related to a constant entropy, may be a feature of the intermediate charged wormhole phase.

we can express the charge as a function of this Green's function,

$$
\begin{aligned}
Q_{a a} & =\frac{1}{N} \sum_{i}^{N}\left\langle\psi_{a i}^{\dagger} \psi_{a i}\right\rangle-\frac{1}{2}=\lim _{\epsilon \rightarrow 0} \frac{1}{N} \sum_{i}^{N}\left\langle\psi_{a i}^{\dagger}(\epsilon) \psi_{a i}(0)\right\rangle-\frac{1}{2}=G_{a a}\left(0^{+}\right)-\frac{1}{2} \\
& =\frac{1}{2}-\frac{1}{N} \sum_{i}^{N}\left\langle\psi_{a i} \psi_{a i}^{\dagger}\right\rangle=\frac{1}{2}-\lim _{\epsilon \rightarrow 0} \frac{1}{N} \sum_{i}^{N}\left\langle\psi_{a i}(0) \psi_{a i}^{\dagger}(-\epsilon)\right\rangle=\frac{1}{2}+G_{a a}\left(0^{-}\right)=\frac{1}{2}-G_{a a}\left(\beta-0^{+}\right),
\end{aligned}
$$

leading to [51]

$$
Q_{a a}=\frac{1}{2}\left(G_{a a}\left(0^{+}\right)-G_{a a}\left(\beta-0^{+}\right)\right) .
$$

Since $G_{L L}(\tau)=G_{R R}(\tau)$ is real, we define the total charge $Q$ as $^{4}$

$Q=Q_{L L}+Q_{R R}=Q_{+}=G_{L L}\left(0^{+}\right)-G_{L L}\left(\beta-0^{+}\right)$.

The temperature dependence of the charge is illustrated in the right column of Fig. 3 for a broad range of parameters. Interestingly, it trails with great accuracy the transition in the grand potential. It is almost temperature independent, and very close to zero, in the low temperature wormhole phase. Physically, it means that at sufficiently low temperatures the wormhole ground state is robust to the presence of a small chemical potential. A finite temperature or chemical potential increases the energy of the system, but the interactions are strong enough to balance these

\footnotetext{
${ }^{4}$ This charge was called $Q_{+}$in the previous sections.
}

increases and keep the charge almost zero. Only the energy gap decreases as $\mu$ increases.

At the two transitions, for small $\kappa$ and $\mu$, the charge jumps, so it can be employed to detect the transition in the system. For larger values of $\kappa$ and $\mu$, the transition in the grand potential becomes a crossover. In this range of parameters, the abrupt discontinuous changes in the charge become sharp but smooth, so the study of $Q$ provides a rather detailed knowledge of the phase diagram of the model.

In Fig. 4 (right column), we show the temperature dependence of $Q$ for a choice of parameters where the two phase transitions are observed with special clarity. In the low temperature phase, the charge is close to zero, but at the low temperature phase transition, it jumps to a finite value. In the intermediate phase, the charge increases approximately linearly with temperature, so in the intermediate region the entropy is constant. At the high temperature phase transition, toward the black hole phase, it jumps again. It remains an open question if the jump in the charge in the low temperature phase transition is associated with a qualitative change in the wormhole ground state; namely, it is unclear whether the wormhole geometry is robust and becomes charged or the finite charge signals a transition to 


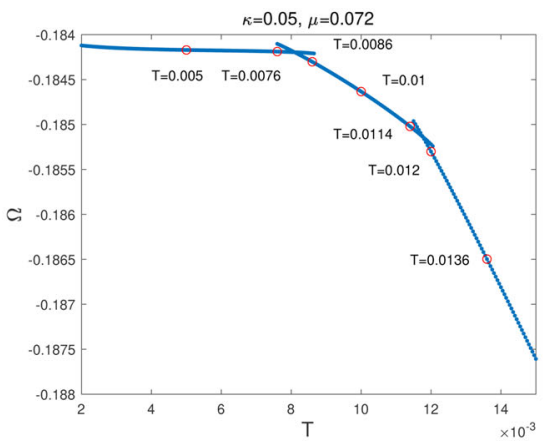

(a)

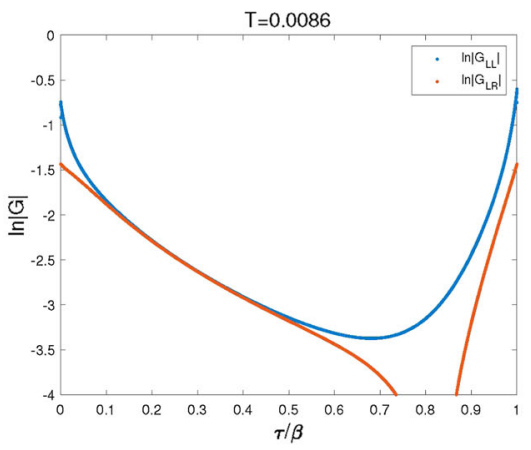

(d)

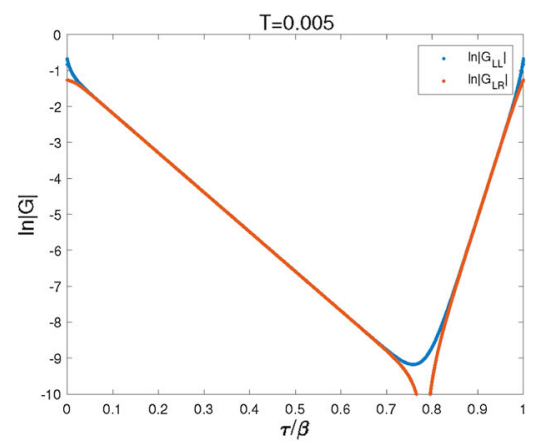

(b)

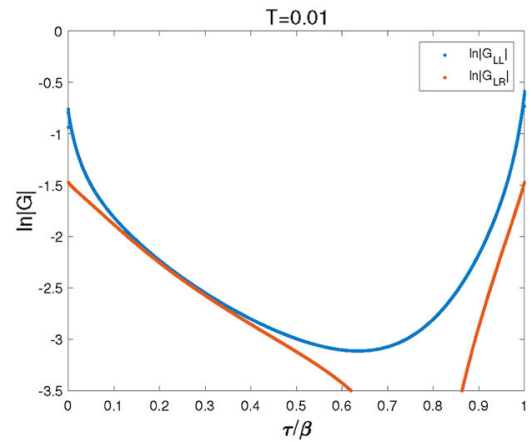

(e)

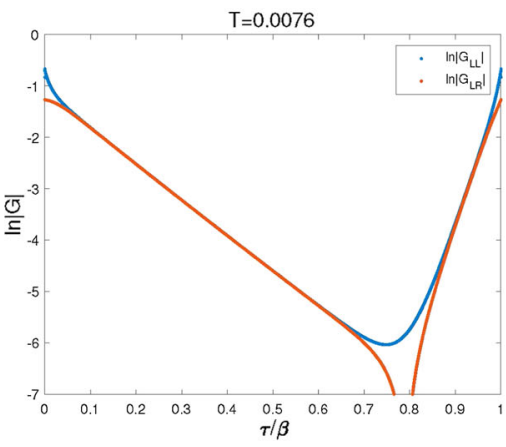

(c)

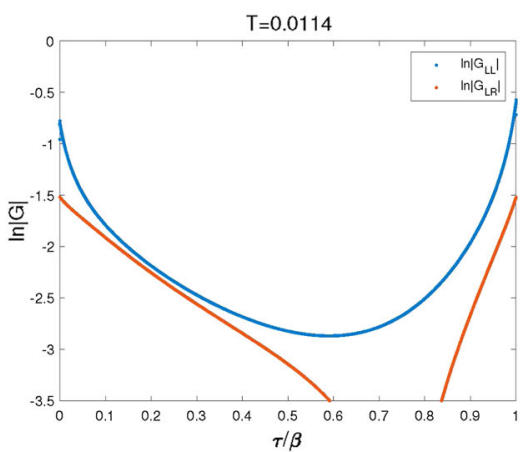

(f)

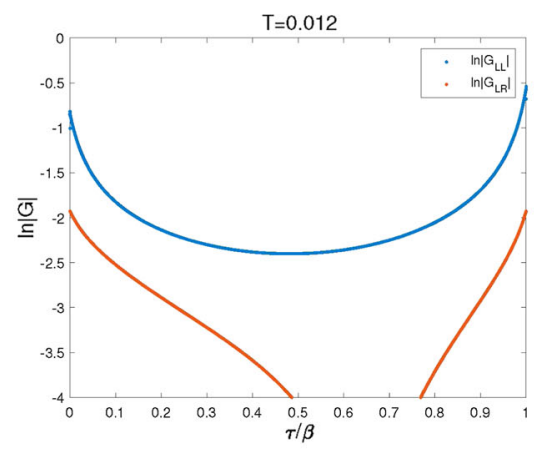

(g)

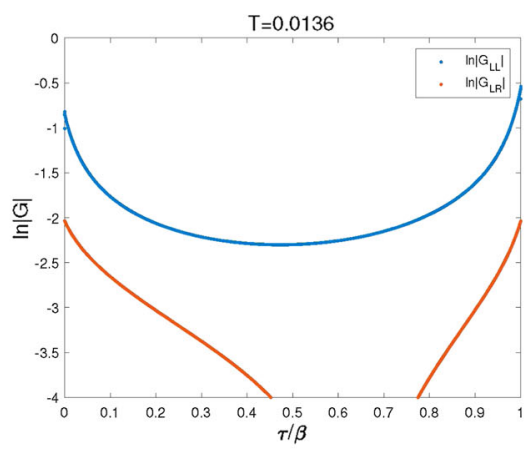

(h)

FIG. 5. Left-top: $\Omega(T)$ for $\mu, \kappa$ in the region of two transitions. The other diagrams represent $\ln G$, with $G=G_{L L}$, $G_{L R}$ vs $\tau / \beta$ in the temperature represented by red circles in $\Omega(T)$. In the intermediate region, especially close to the low temperature transition, the decay is linear, and there is still substantial overlap between $G_{L L}$ and $G_{L R}$, which suggests that some traversability may persist.

a background with no traversability. The calculation of the energy gap will shed some light on this issue.

\section{Energy gap $E_{g}$}

In the case of Majorana fermions, the coupled two-site SYK model [12] has a gap $E_{g}$ in the low temperature limit that is a distinctive feature of the wormhole phase. The almost temperature independence of the grand potential in this limit is a strong indication of a gapped system. More direct evidence of a gap in the spectrum is directly obtained from the decay of the Green's functions. In gapped systems, the decay of $G_{L L}$ or $G_{L R}$ is exponential with a decay rate given by $E_{g}$. Indeed, we shall see $G_{L L}$ and $G_{L R}$ have a similar exponential decay, indicating a similar probability to stay in each site, which implies a continued tunneling between the two sites. This is the type of feature expected in a traversable wormhole. In the low temperature limit, in the region of parameters where we expect a gap, the Green's function is expressed as

$$
G_{a b}(\tau) \sim e^{-E_{g} \tau} f_{a b}(\tau)
$$

with $a=L, R$ and $b=L, R$. Numerically, we shall show that, in this limit, and for sufficiently small $\mu, f_{L L}(\tau) \approx$ $f_{L R}(\tau)$ are nearly constant. We obtain the gap $E_{g}$ by fitting 

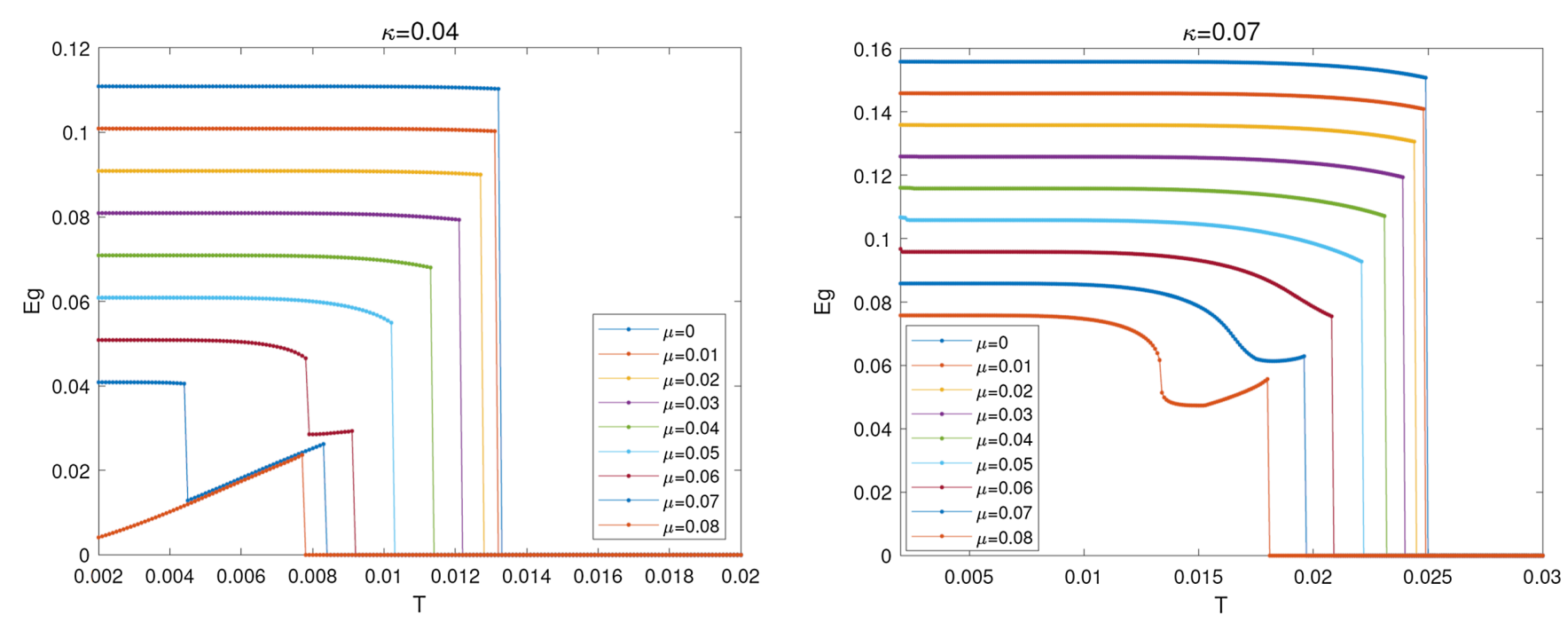

FIG. 6. Summary of the gap $E_{g}$ as a function of $T$ in the region of parameters for which two phase transitions coexist.

the numerical $G_{a b}$ to an exponential. This was also one of the procedures employed for Majorana fermions in Ref. [12] to identify the low temperature phase as a traversable wormhole. In the high temperature limit, we expect a transition, or crossover, to the gapless black hole phase where the ansatz (29) does not apply. A priori, the nature of the decay is unclear in the intermediate phase of finite but small $\mu$.

In Fig. 5, we depict results of the decay of the Green's function in the three phases. We choose parameters, $\kappa=$ 0.05 and $\mu=0.072$, where the existence of two transitions in the grand potential, Fig. 5(a), is more clearly observed. The red circles correspond to the temperatures in which the decay of the Greens's function is studied. As was expected, the Green's function in the low temperature phase, plots (b) and (c) in Fig. 5, decays exponentially, and both Green's functions have a strong overlap. This is the behavior for Majoranas in the wormhole phase [12]. In the high temperature phase, the decays is not exponential for $G_{L R}$, and there is virtually no overlap with $G_{L L}$. This is consistent with a two black hole phase with an explicit coupling between them that does not change the gravitational background.

For intermediate temperatures, between the two phase transitions, the situation becomes more complicated. There is still substantial overlap between $G_{L L}$ and $G_{L R}$, especially close to the low temperature transition. Moreover, the decay is still exponential though in a more limited range of imaginary times which decreases as temperature increases. We tend to believe it is still a wormhole phase but with a worse traversability and, according to previous results, with a finite charge $Q>0$ and constant entropy in this region. However, further calculations are needed to settle the nature of this intermediate region.
In order to gain more explicit information of the gap $E_{g}$, we carry out a fitting of the Green's function by an exponential in a broad range of parameters. Results depicted in the left column of Fig. 3 show that for sufficiently small $\mu$ and not too large $\kappa, E_{g}$ vanishes abruptly at the temperature separating the wormhole from the black hole phase in the same way as in the Majorana case. Only one transition is observed in this region of parameters. As we increase $\mu$, still for small $\kappa$, we access the region where two transitions occur. As was mentioned earlier, the fitting in the intermediate phase becomes less reliable. However, we still observe a sharp drop in $E_{g}$, but it does not vanish at the transition. In the intermediate phase, it increases with temperature, very much like the charge does, and finally vanishes at the higher temperature transition toward the black hole phase. We stress the fitting necessary to obtain $E_{g}$ becomes increasingly unreliable as temperature increases. For instance, the abrupt vanishing of $E_{g}$, see Fig. $3(\mathrm{~g})$, for sufficiently large $\kappa$, is not related to a thermodynamic transition as the grand potential only undergoes a sharp crossover at that temperature.

These results in the intermediate region are confirmed in Fig. 4 (left) for a choice of parameters where the separation between the two transitions is larger. Taking into account that the fitting is more reliable in the lower temperature limit, the existence of a finite gap for temperature slightly above the first transition suggests that the intermediate phase may still be described by a wormhole geometry with limited traversability and a finite charge and constant entropy. However, we stress this is a tentative explanation; it may also occur that a finite charge makes the wormhole phase unstable and the gap in the intermediate phase is not related to a wormhole geometry. However, this intermediate phase is not the high temperature black hole phase in disguise because the grand potential results indicate that 


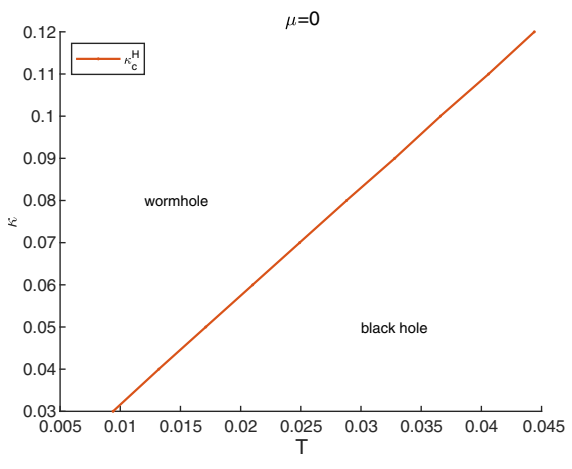

(a)

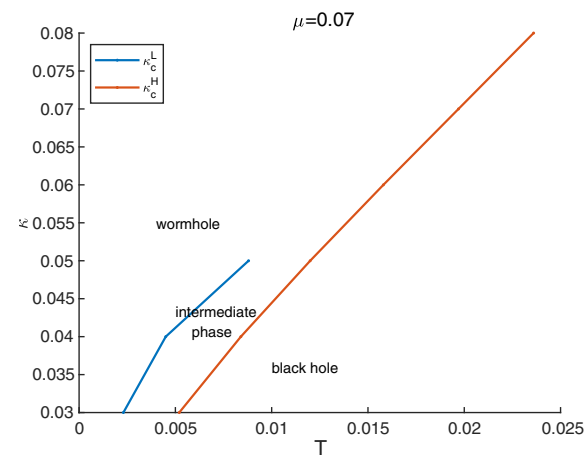

(d)

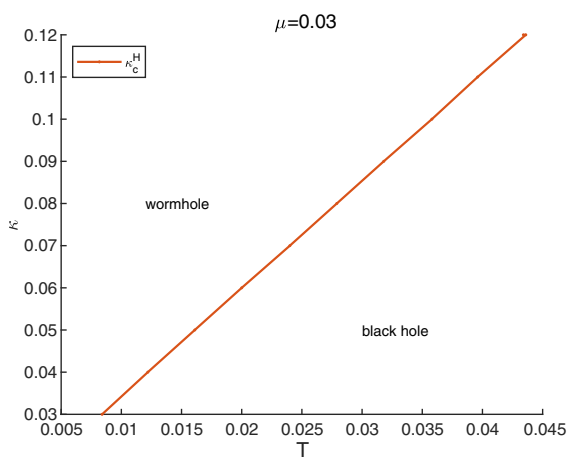

(b)

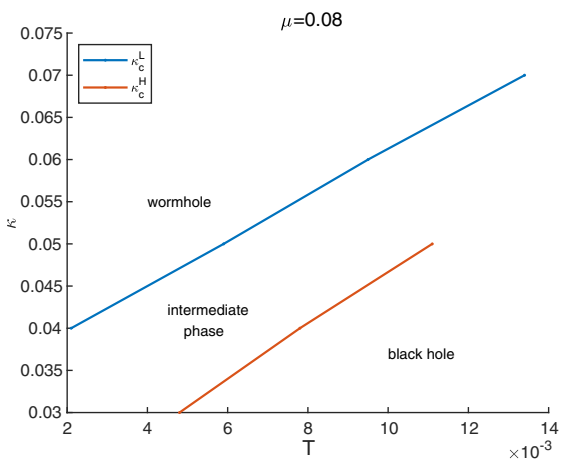

(e)

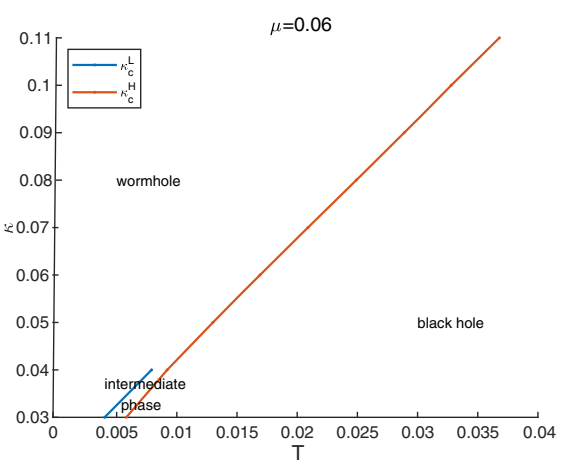

(c)

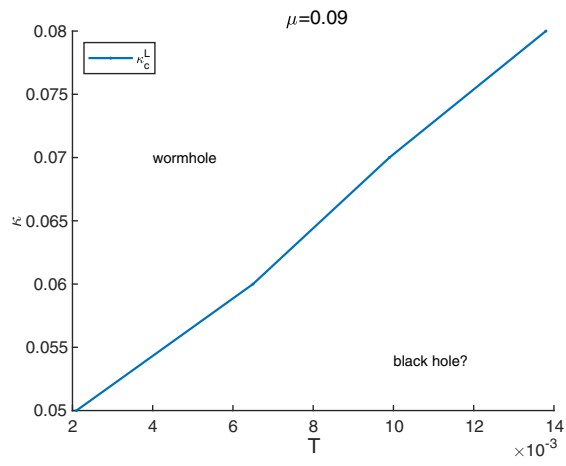

(f)

FIG. 7. Phase diagram of the model as a function of the coupling $\kappa$ and temperature $T$ for different $\mu$ 's obtained from the singularities of the grand potential. Blue (red) lines represent the critical $\kappa$ corresponding to the low (high) temperature phase transition. The end of lines indicates that the phase transition is replaced by a crossover. The intermediate phase, that may correspond to a charged wormhole, is only observed in a limited range of parameters.

this transition occurs at a higher temperature. Therefore, the nature of the intermediate phase, if it is not a charged wormhole, would still be an open question.

Clearly, further calculations are needed to reach a firm conclusion. For the moment, we conclude this section with a summary, see Fig. 6, of the detailed dependence of $E_{g}(T)$ with $\kappa$ and $\mu$ on a broader range of parameters that includes the intermediate region between the two transitions. Results are fully consistent with previous findings for the charge and grand potential.

\section{Phase diagram}

We now combine and extend previous results in order to provide a rather comprehensive description of the system's phase diagram for a given coupling $\kappa$, temperature $T$, and chemical potential $\mu$.

The phase diagram for various $\mu$ 's, extracted from the grand potential, is shown in Fig. 7 as a function of $\kappa$ and temperature. Lines indicate first order phase transitions, and their ends signal that the transition becomes a crossover. For sufficiently small $\mu$, the phase diagram is essentially identical to that of two coupled Majoranas
SYK's where there is a first order phase transition between the wormhole and the black hole phase that becomes a crossover for large $\kappa$.

As $\mu$ increases, the transition occurs at lower temperature. For $\mu \geq 0.06$, and a not too large $\kappa$, we observe two transitions. The one at high temperature corresponds to the transition to the black hole phase. However, there is a new one at lower temperatures which is rather unexpected. Based on the previous results for the charge and the gap, it could in principle be a transition from the wormhole to a charged AdS background with no traversability or to a charged traversable wormhole with limited traversability. As was mentioned earlier, results for the energy gap $E_{q}$ suggest the latter, but more information is needed to clarify this issue. As $\mu$ or $\kappa$ increases further, we shall see the two transitions eventually become crossovers and this intermediate region cease to exist.

In order to gain a further understanding of the phase diagram, we compute the charge $Q$ in the $\kappa-\mu$ parameter space. As is observed in Fig. 8, in the low temperature (left figure), small- $\mu$ limit, the dependence of $\mu$ is very weak, and only one transition is observed, denoted by red circles, between a wormhole phase for larger $\kappa$ and a black hole for 

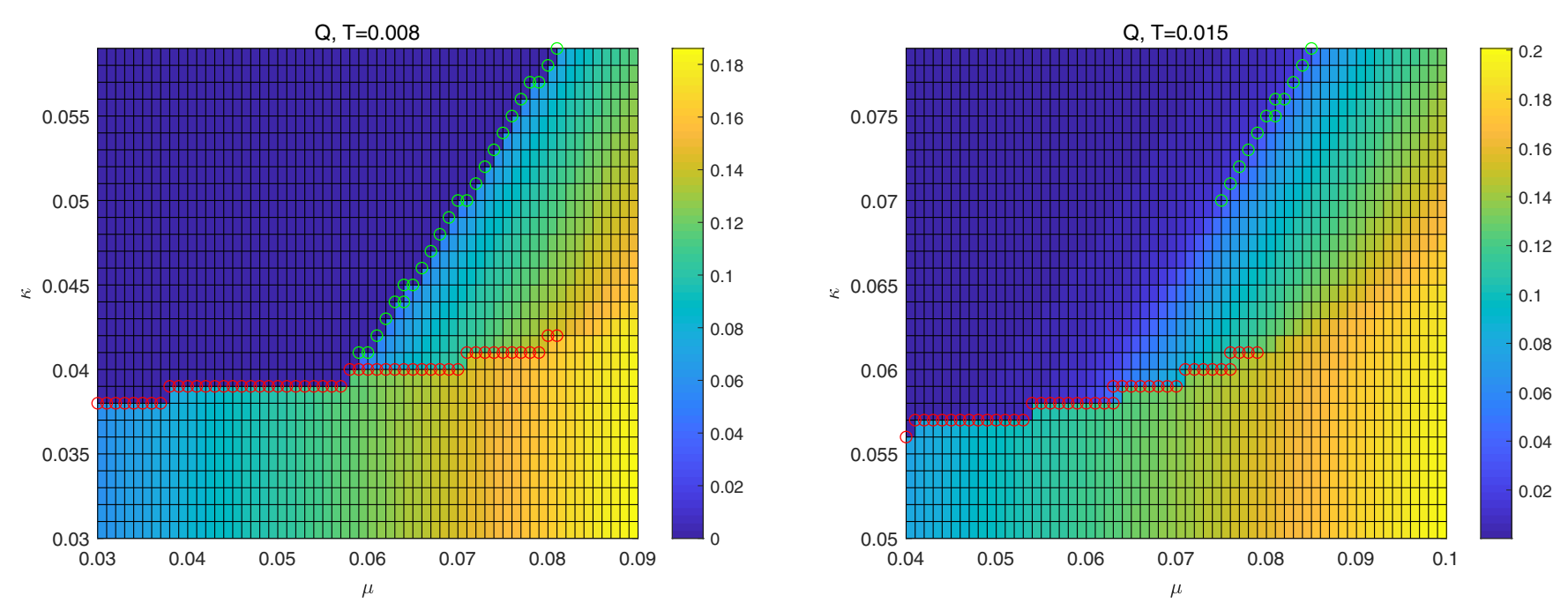

FIG. 8. Charge $Q$ in the $\kappa-\mu$ plane for $T=0.008$ (left) and $T=0.015$ (right). The red circles denote the high temperature wormhole to black hole phase transition, while the green circles denote the novel low temperature phase transition likely between the cold wormhole phase and a phase of limited traversability that may still correspond with a wormhole though with finite charge. Left: temperature is low enough for the intermediate phase between the two phase transitions (light-blue to green region) to be observed. Right: same but for a higher temperature where the low temperature phase transition has turned in a crossover and only the high temperature transition persists.

lower $\kappa$. There is an intermediate region in $\kappa-\mu$ space where the two transitions mentioned above are observed. We note that the one for larger $\kappa$ corresponds to a wormhole phase. The intermediate phase between the two transitions has a finite charge, and as shown earlier, the gap $E_{g}$ is still finite, so it may still be a wormhole but charged and at finite temperature. For sufficiently large $\mu$, the black hole transition becomes a crossover. At sufficiently higher

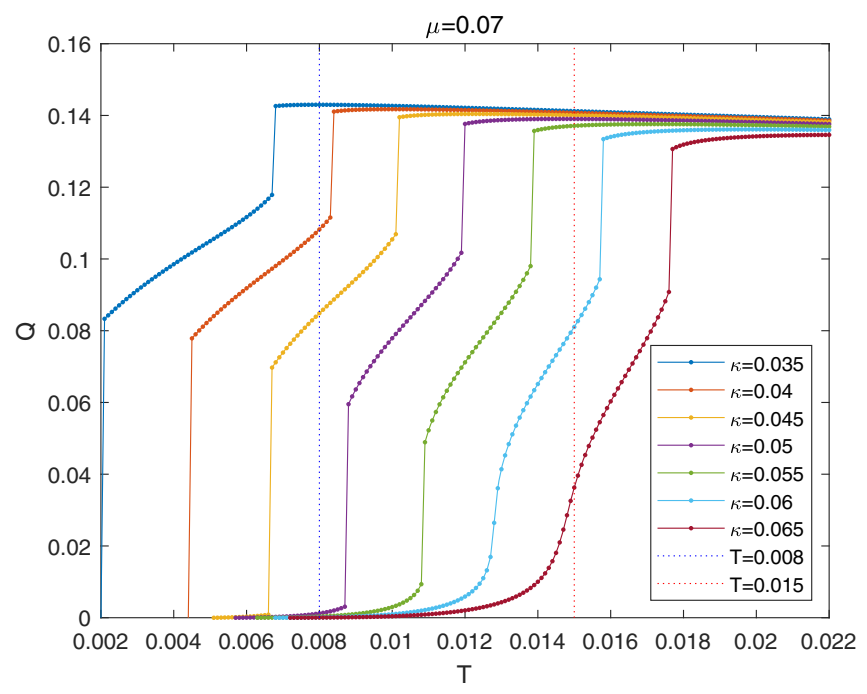

FIG. 9. $Q(T)$ for $\mu=0.07$ with $\kappa=0.035, \ldots, 0.065$. For small $\kappa$, both transitions, characterized by jumps in the charge, are observed. However, as $\kappa$ increases, eventually the low temperature transition becomes a crossover. The vertical lines correspond with the temperatures employed in Fig. 8. As was expected, results of both figures are fully consistent. temperature (right plot), the low temperature phase transition will become crossover in the region of $\mu$ where the high temperature phase transition happens. Therefore, we only observe a transition to a charged black hole. This is fully consistent with the phase diagram obtained from the grand potential.

This picture of the intermediate phase is confirmed explicitly in Fig. 9 for $\mu=0.07$. A fixed and sufficiently low temperature $T=0.008$, represented by a blue dotted vertical line on the left, will intersect the charge curve at couplings $\kappa$ belonging to the black hole, the intermediate, and the wormhole phase in agreement with the results of Fig. 8. Likewise, a red dotted vertical line for a higher temperature will intersect the charge for couplings $\kappa$ that, according to Fig. 8, belong to either the crossover between the chargeless and possibly the charged wormhole or the black hole phase, which confirms that only one transition exists. Eventually, as we further increase $\kappa$, we will see a similar behavior in the high temperature phase transition toward a black hole which will become a crossover.

Finally, for the sake of completeness, we study, see Fig. 10, the gap, the charge, and the grand potential, for larger values of $\kappa$ and $\mu$ and the lowest temperature that we can reach numerically. Interestingly, we observe $E_{g}$ decreases monotonically and almost linearly with $\mu$, for a fixed low $T$ and different values of $\kappa$. It eventually vanishes abruptly for sufficiently large $\mu(\kappa) \sim 0.2$, and it is zero for $\kappa=0$, which suggests $E_{g}$ is related with the wormhole phase and that $\mu$ is only a shift in energy that reduces its value. This is confirmed by the dependence on $\mu$ of the grand potential for $\kappa=0$. This is the only case where 

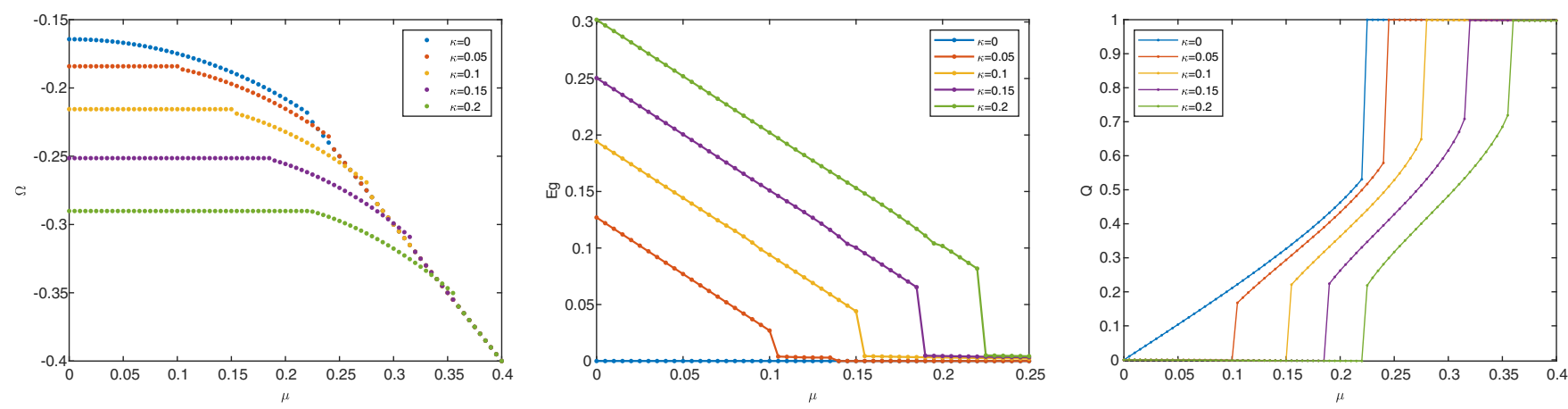

FIG. 10. The grand potential $\Omega$, the charge $Q$, and the gap $E_{g}$ in the low temperature limit for a broad range of parameters $\mu$ and $\kappa$. The transition in the charge for large $Q \geq 0.2$ is not related to the wormhole phase as it also occurs in the single complex SYK [52]. $E_{g}$ decreases monotonically with the chemical potential.

$\Omega$ is not completely flat for small $\mu$, which is an indication that $E_{g}=0$ in this case. For finite $\kappa$, we observe a transition for small $\kappa$ and a crossover for larger $\kappa$.

Another interesting feature is the observed jump in the charge $Q$ for large values of $\mu>0.2$, which are much larger than those corresponding to the transitions studied earlier. Similar results have been reported [52] in single complex SYK, so we do not think that it has any relation with the wormhole phase.

\section{LOW ENERGY EFFECTIVE ACTION}

In this section, we exploit symmetries of the SD equations in the infrared limit in order to find out the low energy effective action of the model.

At low energies $\omega, T \ll J$, the SD equations (21) can be written compactly as

$$
\begin{aligned}
\tilde{\Sigma}_{a b}\left(\tau, \tau^{\prime}\right)= & -(-1)^{q / 2} J^{2} s_{a b}\left[G_{a b}\left(\tau, \tau^{\prime}\right)\right]^{q / 2}\left[G_{b a}\left(\tau^{\prime}, \tau\right)\right]^{q / 2-1} \\
& -\eta_{a b} \delta\left(\tau-\tau^{\prime}\right), \\
& \sum_{b}\left[G_{a b} \star \tilde{\Sigma}_{b c}\right]=-\delta_{a c} \delta\left(\tau-\tau^{\prime}\right),
\end{aligned}
$$

where

$$
\boldsymbol{\eta}_{a b}=\left(\begin{array}{cc}
-\partial_{\tau}+\mu & \eta \\
\eta^{*} & -\partial_{\tau}+\mu
\end{array}\right) .
$$

Ignoring the $\boldsymbol{\eta}_{a b}$ terms in (30a), the above system of SD equations (30) possesses the following time reparametrization and $U(1)$ gauge symmetries,

$$
\begin{aligned}
& G_{a b}\left(\tau, \tau^{\prime}\right) \rightarrow\left[f_{a}^{\prime}(\tau) f_{b}^{\prime}\left(\tau^{\prime}\right)\right]^{\Delta} e^{i\left(\Lambda_{a}(\tau)-\Lambda_{b}\left(\tau^{\prime}\right)\right)} G_{a b}\left(f_{a}^{\prime}(\tau) f_{b}^{\prime}\left(\tau^{\prime}\right)\right), \\
& \tilde{\Sigma}_{a b}\left(\tau, \tau^{\prime}\right) \rightarrow\left[f_{a}^{\prime}(\tau) f_{b}^{\prime}\left(\tau^{\prime}\right)\right]^{1-\Delta} e^{i\left(\Lambda_{a}(\tau)-\Lambda_{b}\left(\tau^{\prime}\right)\right)} \tilde{\Sigma}_{a b}\left(f_{a}^{\prime}(\tau) f_{b}^{\prime}\left(\tau^{\prime}\right)\right),
\end{aligned}
$$

where $f_{a} \in \operatorname{Diff}\left(S^{1}\right), \Lambda_{a} \sim \Lambda_{a}+2 \pi$, and the winding number $n_{a}$ of the compact gauge parameter $\Lambda_{a}$ is conjugate to the $U(1)$ charge $Q_{a}$.

\section{A. High temperature}

Let us first discuss the high temperature limit where we expect that the coupling $\eta$ can be neglected and the SD equations (21) can be solved by an ansatz in which all $L-R, R-L$ functions vanish, describing two copies of a charged SYK model in a thermal state dual to black hole. Standard arguments [39] imply that, at low energies, the effective action of the system is

$$
S=\operatorname{Schw}\left[f_{L}, \Lambda_{L}\right]+\operatorname{Schw}\left[f_{R}, \Lambda_{R}\right],
$$

where the Schwarzian of a single complex SYK model can be expressed $[50,56]$ in terms of the time reparametrization and $U(1)$ gauge symmetries in (32),

$$
\begin{aligned}
\operatorname{Schw}\left[f_{a}, \Lambda_{a}\right]= & -N \alpha_{S} \int d \tau\left\{\tan \frac{f_{a}(\tau)}{2}, \tau\right\} \\
& +\frac{N K}{2} \int d \tau\left(\Lambda_{a}^{\prime}(\tau)+i \mathcal{E}_{a} f_{a}^{\prime}(\tau)\right)^{2},
\end{aligned}
$$

The coefficient $\alpha_{S}$ is the prefactor of the heat capacity in the low temperature limit, $K$ is the compressibility, and $\mathcal{E}$ is an effective parameter that describes the coupling of charge and gravitational degrees of freedom.

The action above has a $[50,58,59] S L(2, \mathbb{R}) \times U(1)$ gauge symmetry. Moreover, there is a global $U(1)$ which will give to a conserved charge.

For a two complex SYK model, at sufficiently high temperature $\beta$, the grand potential is (twice) the usual for the complex SYK model [51],

$$
\frac{\Omega}{2 N}=f\left(\mu_{0}\right)-\mathcal{G}(\mathcal{E}) \beta^{-1}-2 \pi^{2} \alpha_{S} \beta^{-2},
$$

where we have assumed that the two systems are identical. In the above, $f$ is the ground state energy, $\mu_{0}=\mu+2 \pi \mathcal{E} \beta$, 
and $\mathcal{G}(\mathcal{E})$ is the Legendre transform of the entropy $\mathcal{G}(\mathcal{E})=\mathcal{S}(\mathcal{E})-2 \pi \mathcal{E} Q$. The explicit analytical expressions for $\mathcal{E}$ and $Q$ in the case of the single complex SYK model can be found in Ref. [51].

Including small corrections due to the coupling between the two systems can be accounted for perturbatively. We refer to Ref. [12], for a detailed analysis in the case of Majorana fermions.

\section{B. Low temperature}

In the low temperature limit, we expect that the ground state is related to the traversable wormhole. In the Majorana case, it was argued that, in this region, the ground state of the coupled system is close to the TFD state at a particular fictitious temperature $\tilde{\beta}(\eta)[12,45]$ that depends on coupling $\eta$ between sites. We note that the physical temperature is completely unrelated to $\tilde{\beta}(\eta)$.

The $L-R, R-L$ Greens's functions can be obtained simply by analytically continuing the single-sided correlators to complexified time with imaginary part $\tilde{\beta} / 2[60]{ }^{5}$ Additionally, for small enough $\eta$, based on the approximate TFD nature of the ground state [12], we can employ conformal techniques to write down coupling term of the low energy effective action. Analogously, in the gravitational picture, the two spacetime boundaries $(L-R)$ will become causally related in the wormhole phase. In the following, this idea is applied to investigate the coupled complex SYK model.

Based on these assumptions $[18,50,56]$, we can use conformal field theory predictions, also for left-right correlations, together with the effective infrared symmetries of the SD equations mentioned earlier, to write down the Green's functions of our system in the low temperature limit corresponding to the wormhole phase as follows, ${ }^{6}$

$$
\begin{aligned}
& G_{L L}(\tau)=G_{R R}(\tau)=A \frac{e^{-\mathcal{E} \tau}}{\left(\sinh \frac{\tau}{2}\right)^{2 \Delta}} \\
& G_{L R}(\tau)=-G_{R L}(\tau)=B \frac{i e^{-\mathcal{E} \tau}}{\left(\cosh \frac{\tau}{2}\right)^{2 \Delta}}
\end{aligned}
$$

where $A$ and $B$ are undetermined parameters and $0<\tau<\beta$. In the following discussion, we will explore the consistency between results stemming from this ansatz and the previous numerical results.

\footnotetext{
${ }^{5}$ From the dual gravity perspective, this is reflected in the fact that in the complexified maximally extended black hole spacetimes the time on the left part of the wormhole has an imaginary part $\tilde{\beta} / 2$.

${ }^{6}$ Here, we are conventionally setting the period of the thermal circle to $2 \pi$, i.e., $\tau \sim \tau+2 \pi$. After reparametrizing $\tau \rightarrow f(\tau)$, the period will be given by the $\tilde{\beta}$ mentioned below.
}

The reparametrization transformation and gauge transformation in the Green's functions (32),

$$
\left\{\begin{array}{l}
\frac{e^{-\mathcal{E} \tau}}{\left(\sinh \frac{\tau}{2}\right)^{2 \Delta}} \rightarrow e^{i \Lambda(\tau)-\mathcal{E} f(\tau)} \frac{f^{\prime}(\tau)^{\Delta}}{\left(\sinh \frac{f(\tau)}{2}\right)^{2 \Delta}} \\
\frac{i e^{-\mathcal{E} \tau}}{\left(\cosh \frac{\tau}{2}\right)^{2 \Delta}} \rightarrow i e^{i \Lambda(\tau)-\mathcal{E} f(\tau)} \frac{f^{\prime}(\tau)^{\Delta}}{\left(\cosh \frac{f(\tau)}{2}\right)^{2 \Delta}}
\end{array}\right.
$$

leads to the introduction of reparametrization $f(\tau)$ and gauge $\Lambda(\tau)$ modes, which are the effective degree of freedom of the low energy effective action and can be determined by solving the classical equations of motion.

Following the procedure introduced in Ref. [12], see also Ref. [56], we can get the specific form of the Schwarzian in the low energy effective action,

$\begin{aligned} \operatorname{Schw}\left[f_{a}, \Lambda_{a}\right]= & N \alpha_{S} \int d \tau\left\{\tanh \frac{f_{a}(\tau)}{2}, \tau\right\}+\frac{N K}{2} \\ & \times \int d \tau\left(\Lambda_{a}^{\prime}(\tau)+i \mathcal{E} f_{a}^{\prime}(\tau)\right)^{2}, \quad a \in\{L, R\},\end{aligned}$

and the interaction term

$$
\begin{aligned}
S_{\text {int }}= & N \kappa \int d \tau \cos \left(\Lambda_{L}(\tau)-\Lambda_{R}(\tau)\right) \cosh \left(\mathcal{E} f_{L}(\tau)\right. \\
& \left.-\mathcal{E} f_{R}(\tau)\right)\left(\frac{f_{L}^{\prime}(\tau) f_{R}^{\prime}(\tau)}{\cosh ^{2} \frac{f_{L}(\tau)-f_{R}(\tau)}{2}}\right)^{\Delta}
\end{aligned}
$$

As a result, the effective low energy action will be given by

$$
S=\operatorname{Schw}\left[f_{L}, \Lambda_{L}\right]+\operatorname{Schw}\left[f_{R}, \Lambda_{R}\right]+S_{\text {int }},
$$

The low energy solutions (see the Appendix) in the zero and low temperature limits corresponding to the wormhole phase are given by $f(\tau)=f_{L}(\tau)=f_{R}(\tau)=f^{\prime} \tau$ and $\Lambda(\tau)=\Lambda_{L}(\tau)=\Lambda_{R}(\tau)=$ constant

Since the resulting action depends on $\Lambda^{\prime}$ but not on $\Lambda$, there exists a conserved charge,

$$
Q=2 N K\left(-i \Lambda^{\prime}(\tau)+\mathcal{E} f^{\prime}(\tau)\right),
$$

which is indeed the $U(1)$ charge $Q$ computed numerically in the previous section. We recall that numerically we observe that $Q=0$ for sufficiently low temperatures. Given the solutions above, this is only possible provided that $K=0$ in this region. This is indeed plausible taking into account that $K$ has the meaning of compressibility and $Q$ is $\mu$ independent.

The grand potential $\Omega$ is obtained by inserting the proposed solutions for $f$ and $\Lambda$ back in the action, 


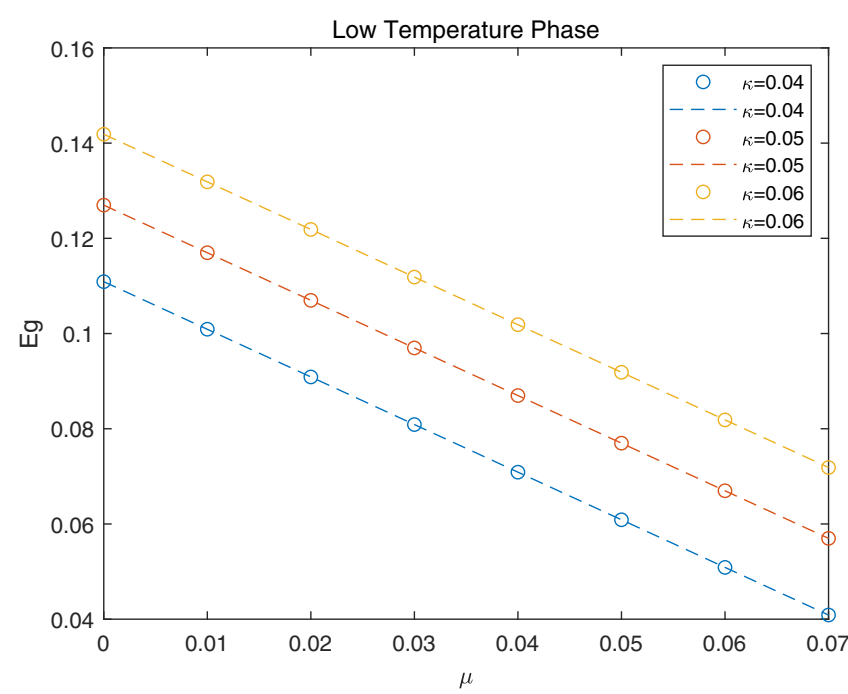

FIG. 11. The gap $E_{g}$ in the low temperature limit as a function of $\mu$ for different $\kappa$ 's. The fitting $E_{g}=E_{0}(\kappa)-\mu$ is in excellent agreement with the numerical results.

$$
\Omega / N=\alpha_{S} f^{\prime 2}+K \mathcal{E}^{2} f^{\prime 2}-\kappa f^{\prime 2 \Delta} .
$$

The value of $f^{\prime}$ can be determined from the charge $Q_{0}$ associated to translations or simply by taking $f^{\prime}$ as a variational parameter and finding the value that minimizes the action [56]. A straightforward calculation shows that

$$
f^{\prime}=\left(\frac{\Delta \kappa}{\alpha_{s}+K \mathcal{E}^{2}}\right)^{\frac{1}{2(1-\Delta)}}
$$

Since $K=0$ in the region for which $Q=0$, we recover the result for the case of Majorana fermions [12], $f^{\prime}=\left(\frac{\Delta \kappa}{\alpha_{s}}\right)^{\frac{1}{2(1-\Delta)}}$.

Finally, let us now find out the prediction for the gap according to the effective low energy effective action. From Eqs. (37), $G_{L L}, G_{L R}$ can be approximated for $\tau \gg \beta$ by

$$
\begin{aligned}
& G_{L L} \sim e^{i \Lambda(\tau)-\mathcal{E} f(\tau)} \frac{f^{\prime}(\tau)^{\Delta}}{\left(\sinh \frac{f(\tau)}{2}\right)^{2 \Delta}} \approx f^{\prime \Delta} e^{-\left(\Delta f^{\prime}+\mathcal{E} f^{\prime}\right) \tau} \\
& G_{L R} \sim i e^{i \Lambda(\tau)-\mathcal{E} f(\tau)} \frac{f^{\prime}(\tau)^{\Delta}}{\left(\cosh \frac{f(\tau)}{2}\right)^{2 \Delta}} \approx i f^{\prime \Delta} e^{-\left(\Delta f^{\prime}+\mathcal{E} f^{\prime}\right) \tau}
\end{aligned}
$$

Numerically, we found in the last section, and in Fig. 11, that for $\tau \gg \beta$

$$
\left|G_{L L}\right| \approx\left|G_{L R}\right| \approx e^{-\left(E_{0}-\mu\right) \tau}
$$

where $E_{0}$ is gap for $\mu=0$, discussed in detail in Ref. [54] for a broad range of $\tau$ 's. The comparison between numerical results and analytical predictions predicts $E_{0}=\Delta f^{\prime}$ and $\mu=-\mathcal{E} f^{\prime}$. The former is indeed the prediction for Majorana fermions [12], and the latter is the expression found for one charged SYK model [56] that agrees with an explicit numerical calculation carried out in the next section.

In summary, we have found agreement between the prediction of the low energy effective action and numerical results in the limit of low temperatures where the $U(1)$ global charge $Q=0$ vanishes. We now address the intermediate region of temperatures where $Q \neq 0$ but the system does not yet seem to be in the black hole phase. First, it is unclear whether the low energy effective action is still applicable or whether it is applicable but the solutions of of the equations of motions employed in the previous case are still valid. However, it seems necessary to impose explicitly the periodicity condition on the solutions $f\left(\tau+\beta_{\mathrm{ph}}\right)=f(\tau)+\beta,\left(\beta=f^{\prime} \beta_{p h}\right)$ and $\Lambda(\tau+\beta)=\Lambda(\tau)$ where $\beta$ is the radius of compactification and $\beta_{p h}$ is the physical temperature. We recall that numerically this intermediate phase is characterized by a finite $Q$ which increases approximately linearly with temperature. Likewise, the gap $E_{g}$ in this region also increases with temperature in a similar fashion, though the fitting is not reliable as the region with an exponential decay of the Green's function is rather narrow. Tentatively, we would expect that if this intermediate phase is still wormholelike the solutions for $f$ and $\Lambda$ employed earlier will still be valid. In that case, the way to proceed is to determine $\beta$ from the minimization of the action [12] and then write $f^{\prime}$ as a function of the critical temperature that can be used to find out the temperature dependence of the charge of the gap $E_{g}$. However, following this procedure, we have not managed to reproduce the numerical results. It could be other solutions for $f$ exists that may explain this intermediate phase or simply that the assumptions made to derive the effective action are no longer applicable in this region. We postpone a detailed study of these issues together with the solution of the associated Liouville quantum mechanical problem and its derivation from a gravity dual to a future publication [61].

\section{Numerical evaluation of $\mathcal{E}$}

In order to gain further insight about the low energy effective action (40), we carry out the numerical evaluation of the parameter $\mathcal{E}$ by fitting the numerical Green's function (36). We restrict ourselves to the wormhole and intermediate phase as this ansatz will work only for sufficiently low temperatures.

In the wormhole phase, we have found that, with great accuracy, $\mathcal{E} f^{\prime} \approx-\mu$, which implies that $E_{g}$ defined in the previous section to characterize the energy gap between the ground state and first excited state in the cold wormhole phase has a simple relation with $\mu, E_{g}=E_{0}-\mu$ where $E_{0}>\mu$ only depends on $\kappa$ and $J$ and therefore it is a more accurate indicator of the wormhole phase. In Fig. 11, we depict the result of the $\mu$ dependence of $E_{g}$ that confirm this simple relation. 
In the intermediate phase, a similar fitting of the Green's function to the ansatz mentioned above points to a relation $-\mathcal{E} f^{\prime} \sim b+c Q \mu$ with $b \sim \mu$ and $c$ a numerical factor of order 1 . However, due to the relatively narrow window of parameters, our results are less reliable than those in the cold wormhole phase, so this expression for $\mathcal{E}$ must be considered more like a conjecture that requires further verification.

In any case, it seems that, as in the canonical ensemble, there is a close relation between $\mathcal{E}$ and the charge $Q$. The presence of a finite chemical potential and charge will reduce the gap induced by the coupling of left and right SYKs but provided that $E_{0}(\kappa)$ is finite and the total gap $E_{g}$ does not vanish, which suggests that the wormhole phase may still exist. In other words, in the intermediate region, the charge jumps to a finite value and then increases linearly with temperature, so this renormalization becomes increasingly important and eventually will destabilize the wormhole phase for sufficiently large $\mu$ or higher temperature. However, if the increase of $Q$ is sufficiently small at the transition, the gap is finite, and the wormhole phase may survive.

Another path to show the existence of the two transitions comes from a qualitative estimation of the critical temperatures from the effective low energy grand potential. At the critical temperature, the grand potential of the two phases must be the same. The black hole high temperature phase is approximately given by two times (35), and the low temperature wormhole phase is given by $E_{g}$. In the intermediate phase, a finite but smaller $E_{g}$ may survive; $Q$ jumps at the two transitions and becomes linear in temperature in between. For the intermediate phase to be a charged wormhole, the zero temperature entropy $\mathcal{S}(\mathcal{E})$ must remain zero. Therefore, for the intermediate phase to be some kind of charged wormhole, we would suggest that the critical temperature of the two phase transitions can be estimated by

$$
\left|E_{g}\right| \sim 4 \pi Q \mathcal{E} f^{\prime} / T, \quad 4 \pi Q \mathcal{E} f^{\prime} / T \sim 2 \mathcal{S}(\mathcal{E}) .
$$

\section{Large- $q$ analysis}

We conclude this section with a large- $q$ analytical analysis of the thermodynamic properties of the system that confirms the existence of the wormhole phase in the low temperature limit. Regarding the intermediate phase, we found solutions with a finite charge and a finite gap, at finite temperature not related to the black hole phase. However, they are observed in a regime where we cannot trust some of the assumptions of the calculation. Therefore, a full analytical characterization of this phase must still be considered an open problem.

The large- $q$ analysis involves the analytical solution of the SD equations by proposing solutions based on an expansion around the free limit $q \rightarrow \infty$ keeping only leading $1 / q$ corrections. There is ample evidence $[12,32,39]$ that most of the interesting properties of the SYK model for $q=4$ are also present in the large- $q$ limit. For the case of a two-site SYK with Majorana fermions, related to traversable wormholes, the large- $q$ expansion is worked out in detail in Ref. [39]. We will adapt this calculation to our two-site complex fermion SYK model with a finite chemical potential. For the sake of simplicity, we will focus on the wormhole region which corresponds to a specific scaling of $q$ with temperature, $\beta \sim q \log q$ in the traversable wormhole case [12].

The strategy of the calculation [12] is to find solutions of the SD equations for small- $\tau \ll q$ and large- $\tau \gg 1$ Euclidean time, both in the large- $q$ limit. These solutions will depend on some unknown parameters that will be fixed by both imposing boundary conditions and matching the solutions at a finite $\tau$. Let us start with the large- $q$ solution in the $\tau \ll q$ limit. Taking into account that we are interested in the wormhole region, we propose the following large- $q$ ansatz:

$$
\left\{\begin{array}{l}
G_{L L}(\tau)=G_{L L}^{0} e^{\frac{1}{q} g_{L L}}=G_{L L}^{0}\left(1+\frac{1}{q} g_{L L}+\cdots\right) \\
G_{L R}(\tau)=G_{L R}^{0} e^{\frac{1}{q} g_{L R}}=G_{L R}^{0}\left(1+\frac{1}{q} g_{L R}+\cdots\right) .
\end{array}\right.
$$

In the free $q \rightarrow \infty$ limit, the solution is given by

$$
G_{L L}^{0}(\tau)= \begin{cases}A e^{\mu \tau}, & \tau>0 \\ -B e^{\mu \tau}, & \tau<0\end{cases}
$$

and

$$
G_{L R}^{0}=i C e^{\mu \tau}
$$

where $A, B$, and $C$ are constants with $A+B=1$ and $\hat{\kappa}=q \kappa$, as in the Majorana SYK case, and $\hat{\mu}=q \mu$. We note that, since $G_{a b}(-\tau)=-G_{a b}(\beta-\tau)$, the above relation also applies in the $\tau \rightarrow \beta$ limit. Moreover, as a consequence of the equation of motion, $G_{L L}=G_{R R}$ and $G_{L R}=-G_{R L}$.

A straightforward calculation shows that $g_{a b}$ verifies the same equations of motion as in the Majorana case [12],

$$
\begin{aligned}
& \partial^{2} g_{L L}(\tau)=2 \mathcal{J}^{2} e^{\frac{1}{2}\left(g_{L L}(\tau)+g_{L L}(-\tau)\right)} \\
& \partial^{2} g_{L R}(\tau)=-2 \mathcal{J}^{2} b^{q-2} e^{\frac{1}{2}\left(g_{L R}(\tau)+g_{L R}(-\tau)\right)}-\frac{\hat{\kappa}}{C} \delta(\tau),
\end{aligned}
$$

with boundary conditions [12],

$$
\begin{aligned}
g_{L L}\left(0^{+}\right) & =g_{L L}\left(0^{-}\right)=0, \\
\partial_{\tau} g_{L R}\left(0^{+}\right)-\partial_{\tau} g_{L R}\left(0^{-}\right) & =-\frac{\hat{\kappa}}{C}
\end{aligned}
$$

and 


$$
g_{L L}(\tau)-g_{L R}(\tau) \rightarrow 0, \quad \tau \rightarrow 0
$$

with parameters

$$
\mathcal{J}^{2}=\frac{q}{2} J^{2}(A B)^{\frac{q}{2}-1}, \quad b=\frac{C}{\sqrt{A B}} .
$$

In the large- $q$ limit, we expect $b=1$, i.e., $C=\sqrt{A B}$.

Therefore, the solutions of (50) are similar to those of the Majorana SYK case [12],

$$
\begin{aligned}
e^{\frac{1}{2}\left(g_{L L}(\tau)+g_{L L}(-\tau)\right)} & =\frac{\alpha^{2}}{\mathcal{J}^{2} \sinh ^{2}(\alpha|\tau|+\gamma)}, \\
e^{\frac{1}{2}\left(g_{L R}(\tau)+g_{L R}(-\tau)\right)} & =\frac{\tilde{\alpha}^{2}}{\mathcal{J}^{2} \cosh ^{2}(\tilde{\alpha}|\tau|+\tilde{\gamma})},
\end{aligned}
$$

which implies

$$
e^{g_{L L}}=\frac{\alpha^{2} e^{\chi(\tau)}}{\mathcal{J}^{2} \sinh ^{2}(\alpha|\tau|+\gamma)}, \quad e^{g_{L R}}=\frac{\tilde{\alpha}^{2} e^{\chi(\tau)}}{\mathcal{J}^{2} \cosh ^{2}(\tilde{\alpha}|\tau|+\tilde{\gamma})},
$$

where $\chi(-\tau)=-\chi(\tau)$.
We follow Ref. [12] and assume $\chi=0$ in the wormhole phase, so

$$
e^{g_{L L}}=\frac{\alpha^{2}}{\mathcal{J}^{2} \sinh ^{2}(\alpha|\tau|+\gamma)}, \quad e^{g_{L R}}=\frac{\tilde{\alpha}^{2}}{\mathcal{J}^{2} \cosh ^{2}(\tilde{\alpha}|\tau|+\tilde{\gamma})}
$$

The constant of integrations are partially determined by the boundary conditions (51) and (52):

$$
\alpha=\mathcal{J} \sinh \gamma, \quad 4 \tilde{\alpha} \tanh \tilde{\gamma}=\frac{\hat{\kappa}}{C} .
$$

It is important to stress that these results assume $\chi(\tau)=0$ and therefore are only valid in the wormhole low temperature region [39]. We cannot rule out that solutions with $\chi(\tau) \neq 0$, that may be related to the intermediate phase, could exist, but we were unable to find them.

We now study the solutions in the opposite infrared limit $\tau \gg 1$. In the low temperature region, $\Sigma_{L R}$ entering in the $\mathrm{SD}$ equations can be approximated [12] by

$$
\Sigma_{L R}(\tau) \sim-i \nu \delta(\tau)
$$

SO

$$
\begin{aligned}
\nu & =i \int d \tau \Sigma_{L R}(\tau) \\
& \left.=\frac{4 C}{q}\left[\tilde{\alpha}+\frac{\mu \tilde{\alpha}}{2 \tilde{\alpha}-\mu}{ }_{2} F_{1}\left(1,1-\frac{\mu}{2 \tilde{\alpha}}, 2-\frac{\mu}{2 \tilde{\alpha}},-e^{-2 \tilde{\gamma})}\right)-\frac{\mu \tilde{\alpha}}{2 \tilde{\alpha}+\mu}{ }_{2} F_{1}\left(1,1+\frac{\mu}{2 \tilde{\alpha}}, 2+\frac{\mu}{2 \tilde{\alpha}},-e^{-2 \tilde{\gamma}}\right)\right)\right] \\
& =\frac{4 C}{q} \tilde{\alpha}+O\left(\frac{\hat{\mu}^{2}}{q^{3}}\right)
\end{aligned}
$$

where we have employed the small- $\tau$ expansion of $\Sigma_{L R}$ to compute $\nu$.

The SD equations are simplified in this limit to

$$
\left(\partial_{\tau}-\mu\right) G_{L L}+i \nu G_{R L}=0, \quad\left(\partial_{\tau}-\mu\right) G_{L R}+i \nu G_{R R}=0
$$

The solutions are given by

$$
\begin{aligned}
& G_{L L}(\tau)=D e^{-(\nu-\mu) \tau}+E e^{-(\nu+\mu)(\beta-\tau)} \\
& G_{L R}(\tau)=F e^{-(\nu-\mu) \tau}-G e^{-(\nu+\mu)(\beta-\tau)}
\end{aligned}
$$

The constants of integrations can be partially fixed by noticing that in low temperature limit

$$
G_{L L}, G_{L R} \sim e^{\mu \tau} e^{ \pm \nu \tau}
$$

which together with the KMS condition $G_{a b}(\tau)=$ $-G_{a b}(\tau+\beta)$, leads to $D \approx F, E \approx G$.

Additional relations are obtained by matching the long and short $\tau$ expansion. In the $q \rightarrow \infty$ limit, $C \approx F \approx G$, $A \approx D, B \approx E$.

Including leading $1 / q$ corrections, $A=\frac{1}{2}-\frac{c}{q}, B=1-$ $A=\frac{1}{2}+\frac{c}{q}$, and $C=\sqrt{\frac{1}{4}-\frac{c^{2}}{q^{2}}}=\frac{1}{2}-\frac{c^{2}}{q^{2}}+\cdots \approx \frac{1}{2}$.

Finally, the explicit matching of the short $\tau$ and long $\tau$ expansion yields $\alpha=\tilde{\alpha}$ and $D=E=F=G=\frac{1}{2}$, as well as $\sigma-c=q e^{-\beta(\nu+\mu)}$ and $\sigma+c=q e^{-\beta(\nu-\mu)}$ from $\tau>0$ and $\tau<0$ regions, respectively. Therefore, we have $\sigma=q e^{-\beta \nu} \cosh (\beta \mu)$ and $c=q e^{-\beta \nu} \sinh (\beta \mu)$, which for $\mu \rightarrow 0$ agrees with the two-site Majorana SYK case. Interestingly, the relation between $\sigma$ and $c$ imposes the restriction $\sigma \geq c$.

From the relations (57) and (59), together with $\alpha=\tilde{\alpha}$ from the matching condition above, we obtain 

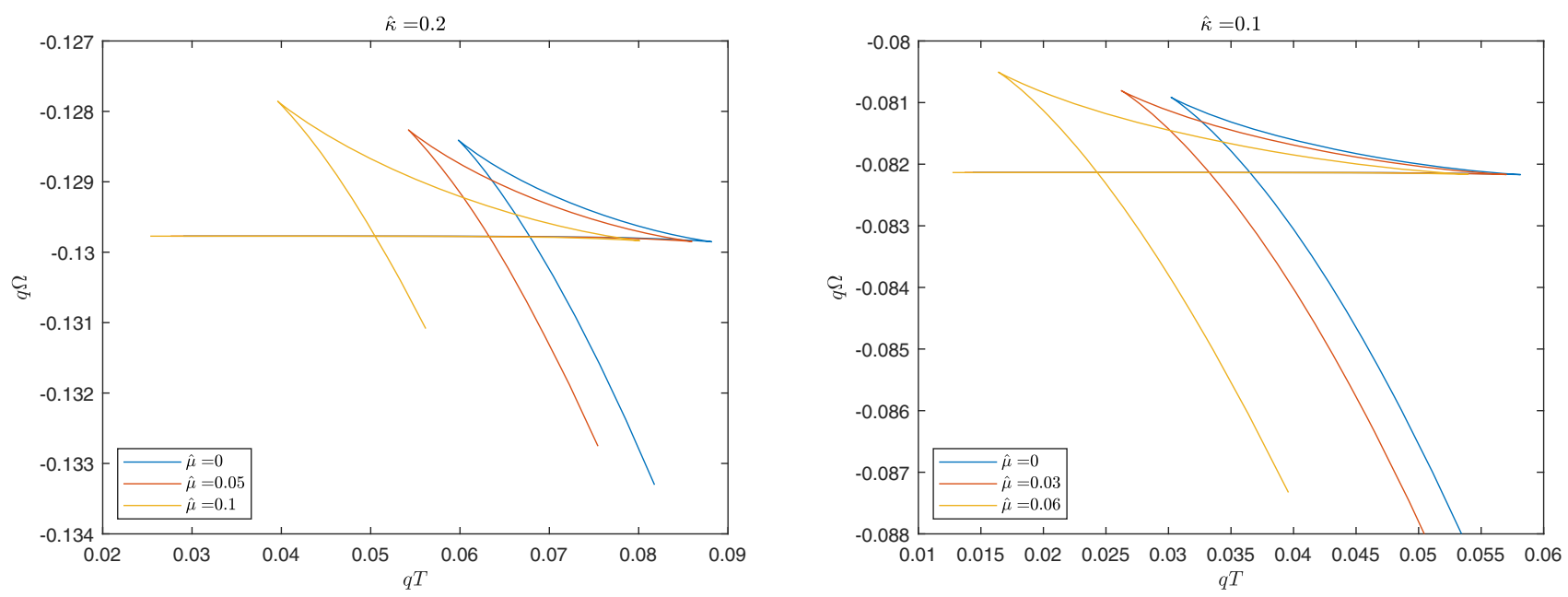

FIG. 12. Left: grand potential $\Omega(64)$ as a function of temperature for $q=56, \hat{\kappa}=0.2, \mathcal{J}=1$ and different $\hat{\mu}^{\prime}$ s. In the low temperature limit, $\Omega$ is constant, in agreement with previous numerical results. Right: the same for a different $\hat{\kappa}=0.1$.

$2 \mathcal{J} \sinh \gamma \tanh \tilde{\gamma}=\frac{\hat{\kappa}}{2 C} \approx \hat{\kappa}$

$$
\log \frac{q}{\sqrt{\sigma^{2}-c^{2}}}=\beta \nu=\frac{\beta \kappa}{\tanh \tilde{\gamma}}, \quad \frac{c}{\sigma}=\tanh \beta \mu .
$$

Following closely the procedure of Ref. [12] for Majorana fermions, we get the grand potential

$$
\begin{aligned}
\beta \Omega= & \frac{\tanh \tilde{\gamma} \log \frac{q}{\sqrt{\sigma^{2}-c^{2}}}}{q}\left(-\frac{1}{\tanh \gamma \tanh \tilde{\gamma}}+1-\frac{q}{2}\right. \\
& \left.-\log \frac{\sinh \gamma}{\cosh \tilde{\gamma}}\right)-\frac{\sigma}{q}\left(1+\log \frac{q}{\sqrt{\sigma^{2}-c^{2}}}\right)
\end{aligned}
$$

and also the total charge and the energy gap,

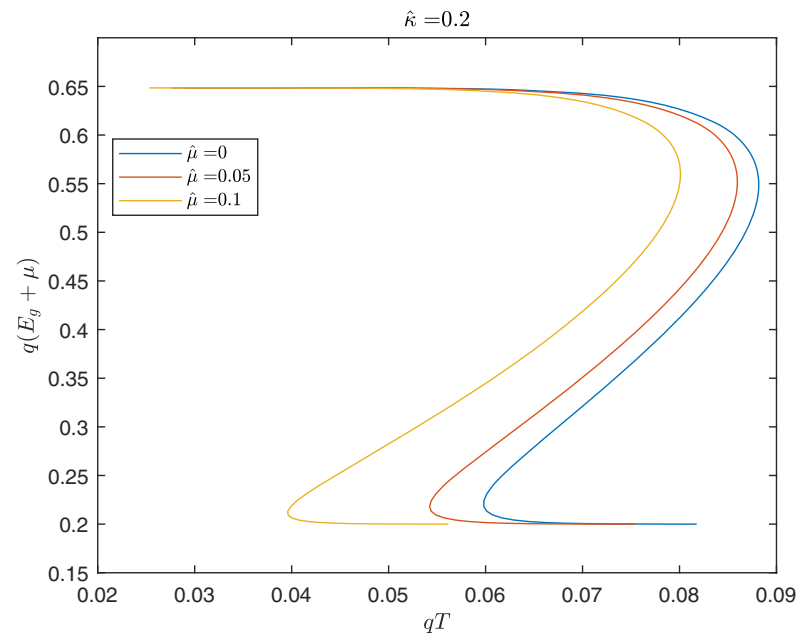

$$
Q=\frac{c}{q}, \quad E_{g}=\frac{2 \alpha}{q}-\mu
$$

We study these quantities as a function of temperature for fixed $\hat{\mu}$ and $\hat{\kappa}$. In the low temperature limit, the results, depicted in Figs. 12-14, are fully consistent with the theoretical expectation and previous numerical results for the wormhole phase. The grand potential $\Omega$ and the gap $E_{g}$ are finite and temperature independent, and the charge $Q$ vanishes. The employed approximations are only valid in this region of very low temperature. For higher temperatures, we observe that, for a given temperature, there are different solutions of the equations. Some of these solutions lead to a finite value of the charge, a sharp drop in the energy gap and a first order transition in the grand potential. These

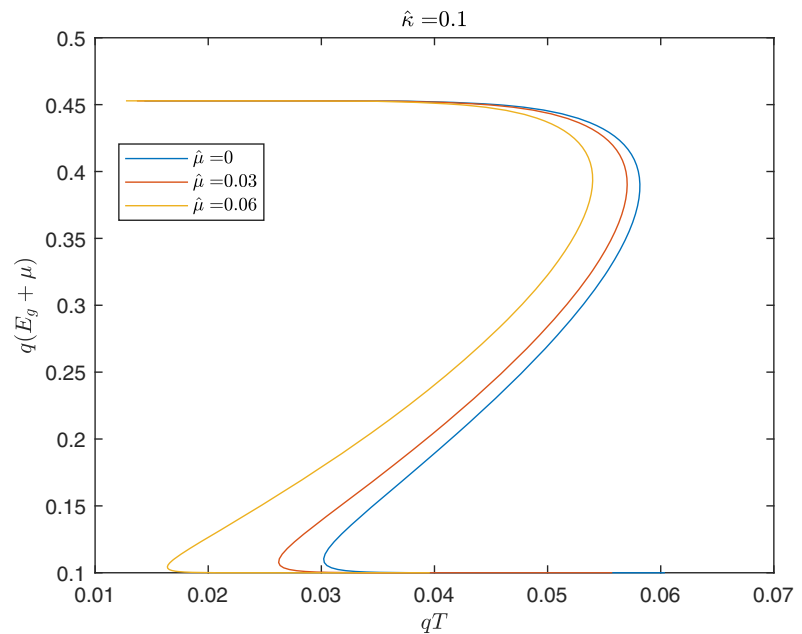

FIG. 13. Left: $E_{g}(65)$ as a function of temperature for $q=56, \hat{\kappa}=0.2, \mathcal{J}=1$ and different $\hat{\mu}$ 's. In agreement with the theoretical expectation, and previous numerical results, $E_{g}$ is almost constant in the low temperature limit, the effect of $\mu$ is a small shift of the gap, and the associated critical temperature though the large- $q$ result breaks down close to the transition. Right: the same for $\hat{\kappa}=0.1$. 

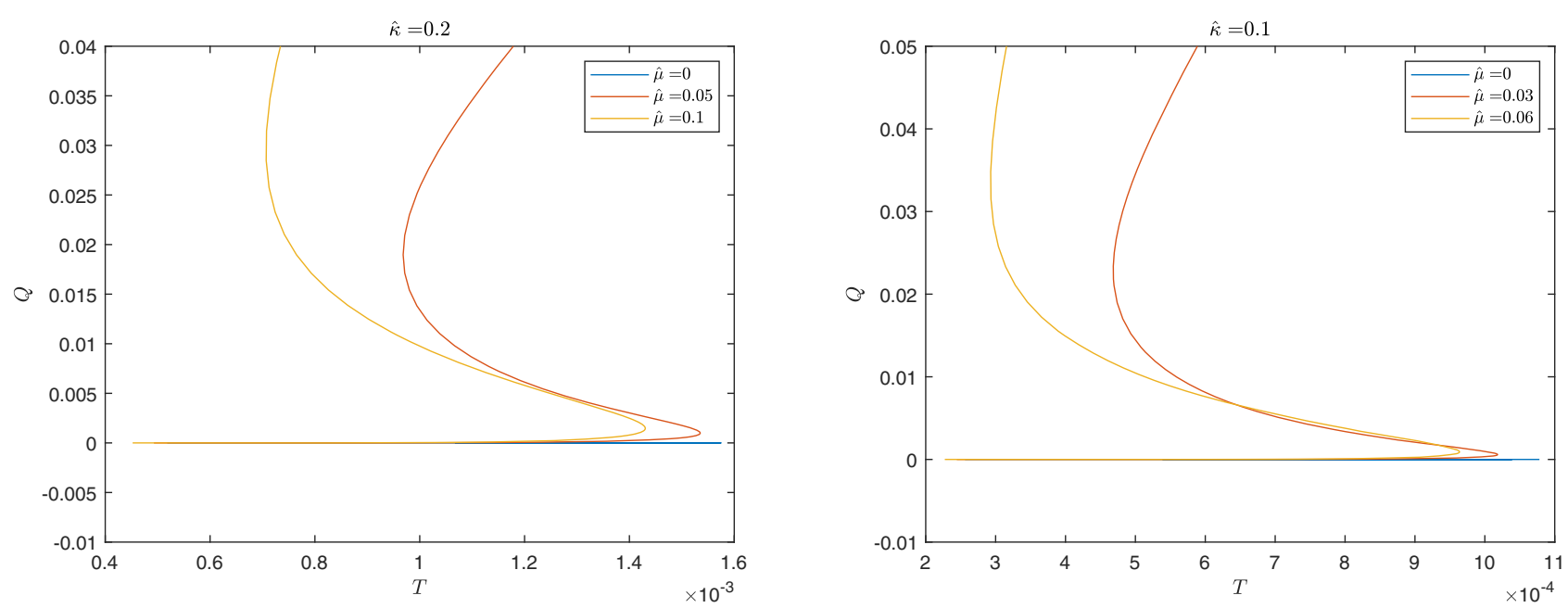

FIG. 14. Left: total charge $Q(65)$ as a function of temperature for $q=56, \hat{\kappa}=0.2, \mathcal{J}=1$ and different $\hat{\mu}=0,0.05,0.1$. In the low temperature region, $Q$ vanishes as is expected in the wormhole phase. Right: the same for $\hat{\kappa}=0.1$.

are features expected in the intermediate phase, which in principle cannot be related to the black hole phase because this requires a different scaling of $q$ with temperature [12]. However, we believe that they do not represent physical solutions that would require for instance a nonvanishing $\chi(\tau)$ partially suppressing the exponential decay of the Green's functions related to the sharp drop of the gap in the intermediate phase. Moreover, according to the numerical results, the approximation for $\Sigma_{L R}$ in (58) breaks down already in this range of temperatures. A new ansatz in place of (58) would modify the effective differential equations in the $\tau \gg 1$ limit, which would lead to further changes in the Green's functions. In summary, in the large- $q$ limit, we have shown analytically the existence of the wormhole phase in the low temperature limit. Further research is needed to clarify whether a genuine wormhole phase can exists at higher temperatures.

\section{CONCLUSIONS}

We have studied a coupled two-site SYK model with Dirac fermions. Many of the features of this model are qualitatively similar to the analogous model with Majorana fermions. For sufficiently small chemical potential, the ground state is gapped with a value that decreases with the chemical potential. It is likely dual to an eternal traversable with zero charge wormhole despite the presence of a finite chemical potential. As temperature increases, and for a small coupling between the two SYKs, eventually we observe a first order transition from the wormhole phase to likely the black hole phase. As the coupling increases, the first order transition eventually becomes a sharp crossover.

As the chemical potential increases, we have found there is an important qualitative difference with respect to the Majorana case: we have identified a range of weak couplings and not too small chemical potentials for which an intermediate phase, tentatively termed the charged wormhole phase, occurs. There is still a gap in the spectrum though the charge, which was zero in the wormhole phase, becomes suddenly finite. It is separated from the black hole phase by a first order transition at higher temperature. At this second critical temperature, the charge undergoes an additional abrupt increase. These transitions become crossovers for sufficiently large chemical potential or strong coupling between the left and right complex SYKs. The thermodynamic features of the model, obtained from the numerical solution of the SD equations, are in qualitative agreement with results obtained from a low energy effective model based on the approximate conformal symmetry of the ground state, close to a charged TFD state. This effective model is a generalized coupled Schwarzian action with extended $S L(2, R) \times U(1)$ symmetry that reflects the additional charge degree of freedom. Finally, we enumerate a few natural extensions of this work. A detailed study of the gravity dual of this model could shed additional light on the nature of the intermediate phase. More specifically, it would be interesting to derive the low energy effective action and the associated Liouville quantum mechanical problem starting from the gravity dual or to extend the novel boundary conditions in $\mathrm{AdS}_{2}$ [62], dual to a single complex SYK, to our coupled complex SYK model. It would also be worthwhile to compute transport properties such as the conductivity in order to further characterize the field theory dual of the wormhole phase. For that, it would also be necessary to generalize the model to higher spatial dimensions. That could bring closer an experimental realization of the physics of the SYK model and its gravity dual. Other venues for further research include the extension of these results to supersymmetric SYK models, nonrandom SYK models, and a detailed description of the real-time formation of a traversable wormhole $[18,63]$. 


\section{ACKNOWLEDGMENTS}

We thank Étienne Lantagne-Hurtubise, author of Ref. [54], for illuminating discussions. We acknowledge financial support from a Shanghai talent program, from the National Natural Science Foundation of China (NSFC) (Grant No. 11874259), and from the National Key R\&D Program of China (Project No. 2019YFA0308603). V. Z. is supported by the China Postdoctoral Science Foundation (International Postdoctoral Fellowship Program 2018).

Note added.-Recently, the paper [54] was posted in the arXiv that investigates the same model, though most of the calculations were focused on the case of zero chemical potential. As far as we know, the intermediate phase was not identified in the model we investigate, though a similar intermediate phase, termed the small black hole phase, was found in a related model. For related work on this model, see also the recent papers $[64,65]$.

\section{APPENDIX: PLAUSIBILITY OF THE $\operatorname{ANSATZ} f_{L}=f_{R}$ AND $\Lambda_{L}=\Lambda_{R}$}

Applying an infinitesimal transformation on $f_{a}$ as in Ref. [12]

$$
\begin{aligned}
& \delta f_{L}=\epsilon_{0}+\epsilon_{+} e^{i f_{L}}+\epsilon_{-} e^{-i f_{L}}, \\
& \delta f_{R}=\epsilon_{0}-\epsilon_{+} e^{i f_{R}}-\epsilon_{-} e^{-i f_{R}}
\end{aligned}
$$

and the transformation for $\Lambda_{a}$,

$$
\delta \Lambda_{a}=-i \mathcal{E} \delta f_{a},
$$

similar to the one copy, the effective action is invariant, and the corresponding Noether charge will be

$$
\begin{aligned}
& Q_{0} / N=Q_{0}\left(f_{L}, \Lambda_{L}\right)+Q_{0}\left(f_{R}, \Lambda_{R}\right)+\left(\frac{1}{f_{L}^{\prime}}+\frac{1}{f_{R}^{\prime}}\right) I_{C} \\
& Q_{+} / N=Q_{+}\left(f_{L}, \Lambda_{L}\right)-Q_{+}\left(f_{R}, \Lambda_{R}\right)+\left(\frac{e^{i f_{L}}}{f_{L}^{\prime}}-\frac{e^{i f_{R}}}{f_{R}^{\prime}}\right) I_{C} \\
& Q_{-} / N=Q_{-}\left(f_{L}, \Lambda_{L}\right)-Q_{-}\left(f_{R}, \Lambda_{R}\right)+\left(\frac{e^{-i f_{L}}}{f_{L}^{\prime}}-\frac{e^{-i f_{R}}}{f_{R}^{\prime}}\right) I_{C}
\end{aligned}
$$

with

$$
\begin{gathered}
Q_{0}(f, \Lambda)=-\alpha_{S}\left(f^{\prime}+\frac{f^{\prime \prime \prime}}{f^{\prime 2}}-\frac{f^{\prime \prime 2}}{f^{\prime 3}}\right) \\
Q_{+}(f, \Lambda)=-\alpha_{S}\left(-i \frac{f^{\prime \prime}}{f^{\prime}}+\frac{f^{\prime \prime \prime}}{f^{\prime 2}}-\frac{f^{\prime \prime 2}}{f^{\prime 3}}\right)
\end{gathered}
$$

$$
\begin{gathered}
Q_{-}(f, \Lambda)=-\alpha_{S}\left(i \frac{f^{\prime \prime}}{f^{\prime}}+\frac{f^{\prime \prime \prime}}{f^{\prime 2}}-\frac{f^{\prime \prime 2}}{f^{\prime 3}}\right) \\
I_{C}=2 \Delta \kappa\left(\frac{f_{L}^{\prime}(u) f_{R}^{\prime}(u)}{\cos ^{2} \frac{f_{L}(u)-f_{R}(u)}{2}}\right) \Delta e^{-\mathcal{E}\left(f_{L}(u)-f_{R}(u)\right)} \\
\times \cos \left(\Lambda_{L}(u)-\Lambda_{R}(u)\right),
\end{gathered}
$$

where $Q_{0}, Q_{+}$, and $Q_{-}$correspond to variations with $\epsilon_{0}, \epsilon_{+}$, and $\epsilon_{-}$, respectively. So, the zero charge condition $Q_{+}=0$ and $Q_{-}=0$ will be satisfied if $f_{L}=f_{R}$. However, it cannot give the constraint on the relation between $\Lambda_{L}$ and $\Lambda_{R}$ since the variation of $\Lambda_{a}$ is not independent. And we need to see whether there is another way to impose the variation to give the constraint.

Assuming $f_{L}=f_{R}$, the invariance of the action under the transformation of $f_{a}$ and $\Lambda_{a}$ requires

$$
\begin{aligned}
& \delta \sum_{a}\left(\Lambda_{a}^{\prime}(\tau)+i \mathcal{E} f_{a}^{\prime}(\tau)\right)^{2} \\
& \quad=2 \sum_{a}\left(\Lambda_{a}^{\prime}(\tau)+i \mathcal{E} f_{a}^{\prime}(\tau)\right)\left(\delta \Lambda_{a}^{\prime}(\tau)++i \mathcal{E} \delta f_{a}^{\prime}(\tau)\right)=0
\end{aligned}
$$

$$
\begin{aligned}
& \delta \cos \left(\Lambda_{L}(\tau)-\Lambda_{R}(\tau)\right) \\
& =-\sin \left(\Lambda_{L}(\tau)-\Lambda_{R}(\tau)\right) \delta\left(\Lambda_{L}(\tau)-\Lambda_{R}(\tau)\right)=0 .
\end{aligned}
$$

These two equation would be satisfied if $\Lambda_{L}=\Lambda_{R}$ and $\delta \Lambda_{L}=-\delta \Lambda_{R}=\epsilon(\tau)$ where $\epsilon(\tau)$ is an arbitrary infinitesimal function. We also need to check whether these relations are consistent with the zero charge condition. As we can see in the former equation above, the charge given by the variation $\delta \Lambda_{L}=-\delta \Lambda_{R}=\epsilon(\tau)$ will be zero trivially, but we still need to check the charge from $\delta f$.

To check the self-consistency of those relation, now we impose the variation

$$
\begin{aligned}
& \delta f_{L}=\epsilon_{0}+\epsilon_{+} e^{i f_{L}}+\epsilon_{-} e^{-i f_{L}}, \\
& \delta f_{R}=\epsilon_{0}-\epsilon_{+} e^{i f_{R}}-\epsilon_{-} e^{-i f_{R}} \quad \delta \Lambda_{L}=-\delta \Lambda_{R}=\epsilon(\tau) ;
\end{aligned}
$$

then, the charge will become

$$
Q_{g} / N=K\left[\left(\Lambda_{L}^{\prime}-\Lambda_{R}^{\prime}\right)+i \mathcal{E}\left(f_{L}^{\prime}-f_{R}^{\prime}\right)\right]
$$

$Q_{0} / N=Q_{0}\left(f_{L}, \Lambda_{L}\right)+Q_{0}\left(f_{R}, \Lambda_{R}\right)+\left(\frac{1}{f_{L}^{\prime}}+\frac{1}{f_{R}^{\prime}}\right) I_{C}$

$$
Q_{+} / N=Q_{+}\left(f_{L}, \Lambda_{L}\right)-Q_{+}\left(f_{R}, \Lambda_{R}\right)+\left(\frac{e^{i f_{L}}}{f_{L}^{\prime}}-\frac{e^{i f_{R}}}{f_{R}^{\prime}}\right) I_{C}
$$




$$
Q_{-} / N=Q_{-}\left(f_{L}, \Lambda_{L}\right)-Q_{-}\left(f_{R}, \Lambda_{R}\right)+\left(\frac{e^{-i f_{L}}}{f_{L}^{\prime}}-\frac{e^{-i f_{R}}}{f_{R}^{\prime}}\right) I_{C}
$$

$$
Q_{-}(f, \Lambda)=e^{-i f}\left[i K \mathcal{E}\left(\Lambda^{\prime}+i \mathcal{E} f^{\prime}\right)-\alpha_{S}\left(i \frac{f^{\prime \prime}}{f^{\prime}}+\frac{f^{\prime \prime \prime}}{f^{\prime 2}}-\frac{f^{\prime \prime 2}}{f^{\prime 3}}\right)\right]
$$

with

$$
\begin{aligned}
& Q_{0}(f, \Lambda)=i K \mathcal{E}\left(\Lambda^{\prime}+i \mathcal{E} f^{\prime}\right)-\alpha_{S}\left(f^{\prime}+\frac{f^{\prime \prime \prime}}{f^{\prime 2}}-\frac{f^{\prime \prime 2}}{f^{\prime 3}}\right) \\
& Q_{+}(f, \Lambda)=e^{i f}\left[i K \mathcal{E}\left(\Lambda^{\prime}+i \mathcal{E} f^{\prime}\right)-\alpha_{S}\left(-i \frac{f^{\prime \prime}}{f^{\prime}}+\frac{f^{\prime \prime \prime}}{f^{\prime 2}}-\frac{f^{\prime \prime 2}}{f^{\prime 3}}\right)\right]
\end{aligned}
$$

$$
\begin{aligned}
I_{C}= & 2 \Delta \kappa\left(\frac{f_{L}^{\prime}(u) f_{R}^{\prime}(u)}{\cos ^{2} \frac{f_{L}(u)-f_{R}(u)}{2}}\right)^{\Delta} e^{-\mathcal{E}\left(f_{L}(u)-f_{R}(u)\right)} \\
& \times \cos \left(\Lambda_{L}(u)-\Lambda_{R}(u)\right)
\end{aligned}
$$

where $Q_{g}, Q_{0}, Q_{+}$, and $Q_{-}$correspond to variations with $\epsilon(\tau), \epsilon_{0}, \epsilon_{+}$, and $\epsilon_{-}$, respectively. Obviously, $t_{L}=t_{R}$, and $\Lambda_{L}=\Lambda_{R}$ is satisfied if imposing $Q_{g}=Q_{+}=Q_{-}=0$. The condition $Q_{0}=0$ is just a consequence that no additional matter is considered in our case [12].
[1] M. S. Morris, K. S. Thorne, and U. Yurtsever, Phys. Rev. Lett. 61, 1446 (1988).

[2] M. Visser, Phys. Rev. D 39, 3182 (1989).

[3] S. W. Hawking, Phys. Rev. D 37, 904 (1988).

[4] M. Visser, Nucl. Phys. B328, 203 (1989).

[5] S. W. Hawking and D. N. Page, Phys. Rev. D 42, 2655 (1990).

[6] P. Gao, D. L. Jafferis, and A. Wall, J. High Energy Phys. 12 (2017) 151.

[7] J. Maldacena, D. Stanford, and Z. Yang, Fortschr. Phys. 65, 1700034 (2017).

[8] Z. Fu, B. Grado-White, and D. Marolf, Classical Quantum Gravity 36, 045006 (2019).

[9] G. Tumurtushaa and D.-H. Yeom, Eur. Phys. J. C 79, 488 (2019).

[10] J. Maldacena, A. Milekhin, and F. Popov, arXiv:1807 .04726 .

[11] A. Anabaln and J. Oliva, J. High Energy Phys. 04 (2019) 106.

[12] J. Maldacena and X.-L. Qi, arXiv:1804.00491.

[13] D. Bak, C. Kim, and S.-H. Yi, J. High Energy Phys. 08 (2018) 140.

[14] W. Israel, Phys. Lett. 57A, 107 (1976).

[15] J. Maldacena, J. High Energy Phys. 04 (2003) 021.

[16] D. Bagrets, A. Altland, and A. Kamenev, Nucl. Phys. B911, 191 (2016).

[17] D. Bagrets, A. Altland, and A. Kamenev, Nucl. Phys. B921, 727 (2017).

[18] J. Maldacena and A. Milekhin, arXiv:1912.03276.

[19] T. Azeyanagi, F. Ferrari, and F. I. S. Massolo, Phys. Rev. Lett. 120, 061602 (2018).

[20] F. Ferrari and F. I. Schaposnik Massolo, Phys. Rev. D 100, 026007 (2019).

[21] J. Kim, I. R. Klebanov, G. Tarnopolsky, and W. Zhao, Phys. Rev. X 9, 021043 (2019).

[22] I. R. Klebanov, A. Milekhin, G. Tarnopolsky, and W. Zhao, J. High Energy Phys. 11 (2020) 162.
[23] O. Bohigas and J. Flores, Phys. Lett. 34B, 261 (1971).

[24] O. Bohigas and J. Flores, Phys. Lett. 35B, 383 (1971).

[25] J. French and S. Wong, Phys. Lett. 33B, 449 (1970).

[26] J. French and S. Wong, Phys. Lett. 35B, 5 (1971).

[27] K. Mon and J. French, Ann. Phys. (N.Y.) 95, 90 (1975).

[28] L. Benet and H. A. Weidenmüller, J. Phys. A 36, 3569 (2003).

[29] V. K. B. Kota, Embedded Random Matrix Ensembles in Quantum Physics (Springer, New York, 2014), Vol. 884.

[30] S. Sachdev and J. Ye, Phys. Rev. Lett. 70, 3339 (1993).

[31] S. Sachdev, Phys. Rev. Lett. 105, 151602 (2010).

[32] A. Kitaev, A simple model of quantum holography, in KITP Strings Seminar and Entanglement 2015 Program, http:// online.kitp.ucsb.edu/online/entangled15/.

[33] W. Fu, D. Gaiotto, J. Maldacena, and S. Sachdev, Phys. Rev. D 95, 026009 (2017).

[34] K. Jensen, Phys. Rev. Lett. 117, 111601 (2016).

[35] A. Jevicki, K. Suzuki, and J. Yoon, J. High Energy Phys. 07 (2016) 1.

[36] J. Maldacena, D. Stanford, and Z. Yang, Prog. Theor. Exp. Phys. 2016, 12C104 (2016).

[37] A. Almheiri and J. Polchinski, J. High Energy Phys. 11 (2015) 1 .

[38] J. Maldacena, S. H. Shenker, and D. Stanford, J. High Energy Phys. 08 (2016) 106.

[39] J. Maldacena and D. Stanford, Phys. Rev. D 94, 106002 (2016).

[40] J. S. Cotler, G. Gur-Ari, M. Hanada, J. Polchinski, P. Saad, S. H. Shenker, D. Stanford, A. Streicher, and M. Tezuka, J. High Energy Phys. 05 (2017) 118.

[41] A. M. García-García and J. J. M. Verbaarschot, Phys. Rev. D 96, 066012 (2017).

[42] A. M. García-García and J. J. M. Verbaarschot, Phys. Rev. D 94, 126010 (2016).

[43] A. Altland and D. Bagrets, Nucl. Phys. B930, 45 (2018).

[44] A. M. García-García, Y. Jia, and J. J. M. Verbaarschot, Phys. Rev. D 97, 106003 (2018). 
[45] A. M. García-García, T. Nosaka, D. Rosa, and J. J. M. Verbaarschot, Phys. Rev. D 100, 026002 (2019).

[46] I. Danshita, M. Hanada, and M. Tezuka, Prog. Theor. Exp. Phys. 2017, 083I01 (2017).

[47] A. Chew, A. Essin, and J. Alicea, Phys. Rev. B 96, 121119 (2017).

[48] D. I. Pikulin and M. Franz, Phys. Rev. X 7, 031006 (2017).

[49] S. Sachdev, Phys. Rev. X 5, 041025 (2015).

[50] R. A. Davison, W. Fu, A. Georges, Y. Gu, K. Jensen, and S. Sachdev, Phys. Rev. B 95, 155131 (2017).

[51] Y. Gu, A. Kitaev, S. Sachdev, and G. Tarnopolsky, J. High Energy Phys. 02 (2020) 157.

[52] N. Sorokhaibam, J. High Energy Phys. 07 (2020) 055.

[53] S. Sachdev, J. Math. Phys. (N.Y.) 60, 052303 (2019).

[54] S. Sahoo, Étienne Lantagne-Hurtubise, S. Plugge, and M. Franz, Phys. Rev. Research 2, 043049 (2020).

[55] W. Fu and S. Sachdev, Phys. Rev. B 94, 035135 (2016).
[56] Y. Gu, A. Kitaev, S. Sachdev, and G. Tarnopolsky, J. High Energy Phys. 02 (2020) 157.

[57] A. Das, Finite Temperature Field Theory (World Scientific, Singapore, 1997).

[58] P. Chaturvedi, Y. Gu, W. Song, and B. Yu, J. High Energy Phys. 12 (2018) 101.

[59] H. R. Afshar, J. High Energy Phys. 02 (2020) 126.

[60] L. Fidkowski, V. Hubeny, M. Kleban, and S. Shenker, J. High Energy Phys. 02 (2004) 014.

[61] A. M. Garcia-Garcia, J. P. Zheng, and V. Ziogas (to be published).

[62] V. Godet and C. Marteau, J. High Energy Phys. 12 (2020) 020.

[63] S. Plugge, E. Lantagne-Hurtubise, and M. Franz, Phys. Rev. Lett. 124, 221601 (2020).

[64] D. L. Nedel, arXiv:2007.06770.

[65] N. Sorokhaibam, arXiv:2007.07169. 